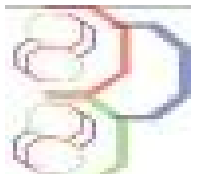

Journal of Applied Biosciences 79:6909-6925

ISSN 1997-5902

\title{
Dynamique du peuplement des Oiseaux d'une riziculture et ses environs dans la zone humide d'importance internationale de Grand-Bassam.
}

\author{
Saint Guillaume K. ODOUKPE, Hilaire K. YAOKOKORE BEIBRO*, Pierre K. KOUADIO et Mickael E. \\ KONAN. \\ Laboratoire de Zoologie et Biologie Animale, UFR Biosciences, Université Félix Houphouët- Boigny d'Abidjan \\ (Côte-d'Ivoire) 22 B.P. 582 Abidjan 22. \\ *Auteur correspondant ; E-mail : hyaokokore@yahoo.fr / Tel +22507634 265
}

Original submitted in on 20 ${ }^{\text {th }}$ April 2014. Published online at www.m.elewa.org on $31^{\text {st }}$ July 2014. http://dx.doi.org/10.4314/jab.v79i1.6

\section{RÉSUMÉ}

Objectifs : cette étude vise à actualiser les connaissances sur l'avifaune de la zone humide de GrandBassam et à déterminer les variations annuelles du peuplement d'Oiseaux.

Méthodologie et résultats : l'avifaune a été inventoriée de janvier 2011 à février 2013 par la méthode des transects itinérants avec des points d'arrêt de 15 minutes aux endroits appropriés. Au total, 218 espèces appartenant à 51 familles et 17 ordres ont été identifiées. La présente étude révèle 49 espèces nouvellement observées dans la zone humide de Grand-Bassam. Le peuplement d'Oiseaux rencontré renferme un grand nombre d'individus dont le pic annuel est atteint en juillet. Ce peuplement est dominé par les populations de tisserin gendarme (Ploceus cucullatus), de travailleur à tête rouge (Quelea erythrops) et de capucin nonnette (Spermestes cucullata).

Conclusion et application : cette étude contribue à une meilleure connaissance de l'avifaune de la zone humide de Grand-Bassam et fournit des données préliminaires sur l'importance numérique des différentes espèces inventoriées. Face à une anthropisation de plus en plus prononcée de la zone humide, liée au développement de la ville et aux activités agricoles, ces résultats serviront de base scientifique à l'établissement d'un système de gestion durable de l'avifaune dans les rizicultures.

Mots clés: Oiseaux, Biodiversité, Dynamique du peuplement, Zone humide Riziculture, GrandBassam.

Dynamics of birds' community, of international importance in the rice fields and surrounding areas in the Grand-Bassam wetland.

Abstract

Objectives: This study aims to update the knowledge on birds' community of the Grand-Bassam wetland and it annual variation.

Methodology and Results: Birds were surveyed from January 2011 to February 2013 by the method of line transects with a 15 minutes points count in the appropriated places. A total 218 species belonging to 51 families of 17 orders were identified. The study found 49 newly observed species in the GrandBassam Ramsar site. The birds' community is rich of an important number of individuals which maximum is in July. This bird's community is dominated by populations of Village Weaver( Ploceus cucullatus),, Red-headed Quelea (Quelea erythrops) and Bronze Mannikin (Spermestes cucullata)..

Conclusion and application: This study has contributed to a better knowledge of the avifauna of GrandBassam Ramsar site and provides preliminary data on the size of the different species. Facing a more 
pronounced anthropisation due to the development of the city and farming, these results can served as a first scientific basis for the establishment of a sustainable management of the avifauna in ricefields.

Keys words: Birds, Biodiversity, Community Dynamics, Wetlands, Ricefield, Grand-Bassam

\section{INTRODUCTION}

La biodiversité est confrontée à une grave crise d'extinction avec des pertes d'espèces qui atteignent des niveaux trois fois plus importants que les moyennes estimées sur les temps géologiques (Brooks et al., 2007). Aussi, des études précises permettant de comprendre les phénomènes qui influencent la distribution des espèces deviennent-elles nécessaires, dans le but notamment de la conservation et de la gestion durable des écosystèmes (Fotso, 1994). On note cependant un déficit important des investissements pour la conservation de la biodiversité (James et al., 1999). En Côte d'Ivoire, en Afrique de l'Ouest, les mesures de conservation de la biodiversité et les moyens financiers qui y sont affectés, concernent, pour la plupart, les écosystèmes forestiers, comme l'attestent de nombreuses études (Walter et al., 1999 ; Yaokokoré-Béibro et Ellenberg, 2000 ; Lachenaud, 2006a, b; Yaokokoré-Béibro, 2010a, b). Pourtant, l'altération de ces écosystèmes forestiers s'accroit considérablement sous l'influence de l'exploitation du bois non durable, de la dégradation forestière, la fragmentation des forêts et de l'augmentation des surfaces cultivées (Yaokokoré-Béibro, 2001, 2010a). Aussi, ces écosystèmes forestiers sont-ils, à eux seuls, loin de pouvoir conserver cette diversité biologique. D'autres écosystèmes tels que les zones humides assurent cette fonction de conservation. Le site Ramsar de Grand-Bassam, en Côte d'Ivoire, fait partie des milieux naturels de conservation de la biodiversité surtout de la

\section{MATÉRIEL ET MÉTHODE}

Site d'étude : Le site d'étude est inclus dans la zone humide de Grand-Bassam et est compris entre $5^{\circ} 13^{\prime} \mathrm{N} / 03^{\circ} 42^{\prime} \mathrm{W}$ et $5^{\circ} 14^{\prime} \mathrm{N} / 3^{\circ} 41^{\prime} \mathrm{W}$. II est aussi délimité naturellement par la lagune Ebrié et le fleuve Comoé. C'est donc une zone marécageuse qui subit, pendant une longue période de l'année, les crues de ces cours d'eau. Le climat est de type tropical humide et caractérisé par deux saisons pluvieuses (avril à mi-juillet et mi-septembre à novembre) et deux saisons sèches (mi-juillet à mi-septembre et diversité de l'avifaune. Des études ornithologiques préliminaires attestent bien la richesse de l'avifaune de cette zone humide et révèlent la présence d'espèces inscrites sur la liste rouge de I'UICN telles que le faucon crécerelette Falco naumanni (Fleischer, 1818), le perroquet jaco Psittacus erithacus Linné 1758, la sterne des baleiniers Sterna balaenarum (Strickland, 1852), (Odoukpé, 2010 ; YaokokoréBéibro et al., 2010 ; Gueye, 2013). Cependant, la protection des écosystèmes et la préservation des espèces vivantes en danger exigent la mise en œuvre de mesures de conservation judicieuses, fondées sur des bases scientifiques bien établies (Ramade, 2005); aussi la zone humide de Grand-Bassam a-t-elle été classée, selon les critères de la convention de Ramsar, zone humide d'importance internationale (Yaokokoré-Béibro et al., 2005 ; Secrétariat de la convention de Ramsar, 2010). Cette zone humide bénéficie à ce titre de la protection et des mesures de conservation pour les Oiseaux telles que préconisées par ladite convention (Secrétariat de la convention de Ramsar, 2006). Ainsi, pour mettre en place un système de gestion durable de cet écosystème, la connaissance approfondie de l'avifaune s'avère nécessaire. En cela, une étude ornithologique qui vise à actualiser les connaissances sur l'avifaune de la zone humide de Grand-Bassam ainsi qu'à déterminer les variations du peuplement d'Oiseaux a été initiée dans une riziculture et ses environs durant deux années.

décembre à mars) (Brou, 1997). La pluviométrie moyenne annuelle est de $2100 \mathrm{~mm}$ avec une température moyenne annuelle de $26,5^{\circ} \mathrm{C}$ et une humidité relative avoisinant $85 \%$. La région appartient au secteur littoral du grand domaine phytogéographique guinéen et est caractérisée par divers types de végétations marécageuses (Guillaumet et Adjanohoun, 1971).

Matériel technique : Le matériel d'observation se compose de paires de jumelles pour l'observation 
directe des Oiseaux. Le matériel d'enregistrement est représenté par des carnets de note pour la retranscription des relevés et un appareil photographique numérique reflexe (NIKON D5000, objectif: $18-200 \mathrm{~m}$ ) pour les prises de vue. Le matériel d'identification se compose de guides d'identification des Oiseaux de l'Afrique de l'ouest (Serle et Morel, 1993 ; Borrow et Demey, 2008) et de disques compacts des chants et cris des Oiseaux d'Afrique (Chapuis, 2000). Le matériel de géolocalisation est un appareil GPS (Map source Garmin GPS 60cs) pour la détermination des coordonnées géographiques du site d'étude.

Collecte des données: II est assez difficile de définir une méthode standard pour le dénombrement des populations d'Oiseaux dans un milieu quelconque. En général, la méthode adoptée pour un dénombrement dépend des objectifs visés par l'étude, des conditions d'application et aussi des aptitudes de l'auteur (Bibby et al., 1992). Ainsi, pour les besoins d'inventaires réguliers mensuels, seuls les habitats accessibles et praticables sur toute l'année ont été retenus. De ce fait, l'ensemble des habitats retenus a été scindé en deux zones distinctes qui ont fait chacune l'objet d'inventaire. Dans chaque zone, quatre inventaires ornithologiques sont effectués tous les mois de janvier 2011 à février 2013. Tous les Oiseaux vus ou entendus posés ou au vol à l'intérieur de la zone considérée sont notés de $06 \mathrm{~h} 30$ à $10 \mathrm{~h} 30$ et $16 \mathrm{~h} 00$ à 18h00. Le dénombrement s'est fait par individu ou par estimation de groupe de 10 ou 100 lorsque ceuxci étaient en grand nombre. La méthode des transects itinérants avec des points d'arrêt de 15

\section{RÉSULTATS}

Composition spécifique des Oiseaux:, La richesse avifaunistique de la zone de riziculture et ses environs est estimée à 218 espèces appartenant à 51 familles et regroupées en 17 ordres (Tableau I). Les Non-Passériformes avec 126 espèces de 32 familles et 16 ordres représentent $57,80 \%$ du peuplement. L'ordre des Charadriiformes avec 22 espèces regroupées dans cinq familles est le mieux représenté. L'ordre des Falconiformes suit avec 18 esp de trois familles. Dans ce même groupe taxonomique, les familles les mieux représentées minutes aux endroits appropriés a été retenue (Yaokokoré-Béibro et al., 2010). Pour limiter les erreurs d'analyse des paramètres de l'avifaune, il sera exclu les populations des rapaces nocturnes et celles des espèces rencontrées en dehors des sessions de dénombrement. Cependant, ces espèces compteront pour la richesse de la faune avienne du site.

La liste des espèces est faite selon l'ordre phylogénétique de Borrow et Demey (2001). Le statut biogéographique (Borrow et Demey, 2008), l'habitat préférentiel (Bennun et al., 1996 ; Yaokokoré-Béibro, 2001) et le biome (Fishpool et Evans, 2001) de chaque espèce sont indiqués. La valeur maximale (IPA) des quatre inventaires mensuels par zone est retenue pour les différentes analyses statistiques. La fréquence relative $(\mathrm{Fr})$, l'indice de diversité de Shannon-Wiener $(\mathrm{H}$ ') et l'indice d'équitabilité $(\mathrm{J})$ ont été calculés à l'aide du logiciel Microsoft Excel 2010.

$\mathrm{Fr}=\mathrm{n}_{\mathrm{i}} / \mathrm{N}$ avec $\mathrm{n}_{\mathrm{i}}$ : somme de tous les contacts avec l'espèce i à chaque stade de dénombrement ; $\mathbf{N}: \sum n_{i}$ : somme des contacts avec l'ensemble des espèces observées à chaque stade.

$\mathbf{H}^{\prime}=-\sum\left(\mathbf{n}_{\mathbf{i}} / \mathbf{N}\right) \ln \left(\mathrm{n}_{\mathrm{i}} / \mathbf{N}\right)$ (Ramade, 1994)avec In : logarithme népérien ;

$\mathrm{J}=\mathrm{H}^{\prime} / \mathrm{H}$ 'max Avec H'max : valeur maximale de $\mathrm{H}^{\prime}$ et $\mathrm{H}^{\prime} \quad \max =\ln$ (nombre d'espèces).

Pour caractériser les différentes espèces observées, sur la base de leur population, les normes selon Thiollay (1986) qui tiennent compte de la fréquence relative (Fr) d'observation de l'espèce ont été utilisées. L'analyse de variance a été effectuée au seuil de 5\% à l'aide du logiciel STATISTICA 7.1

sont celles des Ardeidae, Accipitridae et Scolopacidae avec 13 espèces chacune. L'ordre des Passériformes compte à lui seul 92 espèces appartenant à 19 familles et représente $42,20 \%$ du peuplement. Les familles les mieux représentées sont celles des Sylviidae et des Pycnonotidae avec respectivement 17 espèces et 12 espèces. La figure 1 présente les images de quelques espèces d'Oiseaux rencontrées dans la zone humide d'importance internationale de Grand-Bassam. 
Tableau 1: Bilan des espèces d'Oiseaux observées de janvier 2011 à Février 2013

\begin{tabular}{|c|c|c|c|c|c|c|c|c|c|c|c|c|c|c|c|c|c|c|c|}
\hline ORDRES /Familles/Espèces & Bio & $\mathrm{Hab}$ & Jan & Fév & Mars & Avr & Mai & Juin & Juil & Août & Sept & Oct & Nov & Déc & IPA & $\begin{array}{c}\text { Moy } \\
\text { Men士Ec-Ty }\end{array}$ & EC & $\mathrm{Fr}$ & IA \\
\hline $\begin{array}{l}\text { PODICIPEDIFORMES } \\
\text { Podicipedidae }\end{array}$ & & & & & & & & & & & & & & & & & & & \\
\hline $\begin{array}{l}\text { Tachybaptus ruficollis (Pallas, 1764) } \\
\text { PELECANIFORMES } \\
\text { Phalacrocoracidae }\end{array}$ & $\mathrm{R}$ & $\mathrm{E}$ & 0 & 0 & 0 & 0 & 0 & 0 & 0 & 0 & 0 & 2 & 1 & 0 & 2 & $0,25 \pm 0,62$ & 3 & 0 & $A c$ \\
\hline $\begin{array}{l}\text { Phalacrocorax africanus (J. F. Gmelin, 1789) } \\
\text { Anhingide } \\
\text { Anhinga rufa (Daudin, 1802) }\end{array}$ & $\begin{array}{l}\mathrm{R} \\
\mathrm{R}\end{array}$ & $\begin{array}{l}E \\
E\end{array}$ & 17 & 11 & 40 & 12 & 21 & 34 & 33 & 28 & 30 & 45 & 58 & 8 & 58 & $28,08 \pm 15,11$ & 337 & 0,19 & Ac \\
\hline $\begin{array}{l}\text { CICONIIFORMES } \\
\text { Ardeidae }\end{array}$ & & & & & & & & & & & & & & & & & & & \\
\hline Ixobrychus minutus (Linné, 1766) & $R / P$ & $\mathrm{E}$ & 0 & 0 & 2 & 0 & 7 & 13 & 14 & 8 & 2 & 3 & 4 & 1 & 14 & $4,5 \pm 4,95$ & 54 & 0,03 & Ac \\
\hline Nycticorax nycticorax (Linné, 1758) & $\mathrm{R} / \mathrm{P}$ & $\mathrm{E}$ & 15 & 18 & 12 & 23 & 47 & 93 & 144 & 69 & 11 & 17 & 34 & 28 & 144 & $42,58 \pm 40,64$ & 511 & 0,29 & $\mathrm{Ra}$ \\
\hline Ardeola ralloides (Scopoli, 1769) & $\mathrm{R} / \mathrm{P}$ & $\bar{E}$ & 24 & 23 & 11 & 25 & 89 & 48 & 35 & 24 & 20 & 38 & 27 & 13 & 89 & $31,42 \pm 20,83$ & 377 & 0,22 & $\mathrm{Ra}$ \\
\hline Bubulcus ibis (Linné, 1758) & $\mathrm{R} / \mathrm{M}$ & $\mathrm{E}$ & 439 & 332 & 283 & 227 & 251 & 214 & 221 & 117 & 196 & 313 & 270 & 247 & 439 & $259,17 \pm 79,87$ & 3110 & 1,78 & $\mathrm{R}$ \\
\hline Butorides striata (Linné, 1758) & $\mathrm{R}$ & $\mathrm{E}$ & 3 & 0 & 0 & 2 & 7 & 6 & 9 & 8 & 3 & 6 & 6 & 5 & 9 & $4,58 \pm 2,97$ & 55 & 0,03 & Ac \\
\hline Egretta ardesiaca (Wagler, 1827) & $\mathrm{R} / \mathrm{M}$ & $\mathrm{E}$ & 0 & 0 & 0 & 0 & 0 & 0 & 0 & 0 & 0 & 0 & 1 & 0 & 1 & $0,08 \pm 0,29$ & 1 & 0 & Ac \\
\hline Egretta gularis (Bosc, 1792) & $\mathrm{R}$ & $\mathrm{E}$ & 1 & 2 & 1 & 2 & 6 & 2 & 1 & 0 & 2 & 9 & 4 & 2 & 9 & $2,67 \pm 2,53$ & 32 & 0,02 & Ac \\
\hline Egretta garzetta (Linné, 1766) & $\mathrm{R} / \mathrm{M}$ & $\mathrm{E}$ & 8 & 5 & 2 & 13 & 15 & 5 & 1 & 0 & 1 & 2 & 9 & 7 & 15 & $5,67 \pm 4,89$ & 68 & 0,04 & Ac \\
\hline Egretta intermedia (Wagler, 1829) & $\mathrm{R} / \mathrm{M}$ & $\mathrm{E}$ & 7 & 5 & 14 & 36 & 30 & 16 & 6 & 7 & 12 & 24 & 32 & 10 & 36 & $16,58 \pm 11,08$ & 199 & 0,11 & Ac \\
\hline Egretta alba (Linné, 1758) & $\mathrm{R} / \mathrm{M}$ & $\mathrm{E}$ & 9 & 8 & 36 & 48 & 56 & 30 & 11 & 24 & 83 & 60 & 25 & 24 & 83 & $34,5 \pm 23,13$ & 414 & 0,24 & $\mathrm{Ra}$ \\
\hline Ardea purpurea Linné, 1766 & $\mathrm{R} / \mathrm{P}$ & $\bar{E}$ & 7 & 4 & 4 & 5 & 5 & 19 & 5 & 4 & 2 & 9 & 4 & 4 & 19 & $6 \pm 4,45$ & 72 & 0,04 & Ac \\
\hline Ardea cinerea Linné, 1758 & $\mathrm{R} / \mathrm{P}$ & $\mathrm{E}$ & 3 & 2 & 0 & 0 & 0 & 0 & 2 & 0 & 1 & 3 & 3 & 2 & 3 & $1,33 \pm 1,3$ & 16 & 0,01 & Ac \\
\hline $\begin{array}{l}\text { Ardea melanocephala Vigors \& Children, } 1826 \\
\text { Ciconiidae }\end{array}$ & $\mathrm{R} / \mathrm{M}$ & $\mathrm{E}$ & 0 & 1 & 3 & 0 & 1 & 0 & 0 & 0 & 0 & 0 & 0 & 0 & 3 & $0,42 \pm 0,9$ & 5 & 0 & Ac \\
\hline Anastomus lamelligerus Temminck, 1823 & M & $E$ & 0 & 0 & 0 & 0 & 1 & 0 & 0 & 0 & 0 & 0 & 0 & 0 & 1 & $0,08 \pm 0,29$ & 1 & 0 & Ac \\
\hline $\begin{array}{l}\text { Ciconia episcopus (Boddaert, 1783) } \\
\text { Threskiornithidae }\end{array}$ & $\mathrm{R} / \mathrm{M}$ & $\mathrm{E}$ & 3 & 1 & 4 & 6 & 5 & 0 & 1 & 0 & 0 & 3 & 3 & 0 & 6 & $2,17 \pm 2,12$ & 26 & 0,01 & Ac \\
\hline Plegadis falcinellus (Linné, 1766) & $\mathrm{R} / \mathrm{P}$ & $E$ & 0 & 0 & 0 & 0 & 2 & 0 & 0 & 0 & 0 & 1 & 0 & 0 & 2 & $0,25 \pm 0,62$ & 3 & 0 & Ac \\
\hline $\begin{array}{l}\text { ANSERIFORMES } \\
\text { Anatidae }\end{array}$ & & & & & & & & & & & & & & & & & & & \\
\hline Dendrocygna viduata (Linné, 1766) & $\mathrm{R} / \mathrm{M}$ & $\mathrm{E}$ & 262 & 284 & 98 & 175 & 500 & 121 & 97 & 110 & 37 & 190 & 387 & 97 & 500 & $196,5 \pm 137,83$ & 2358 & 1,35 & $\mathrm{R}$ \\
\hline Plectropterus gambensis (Linné, 1766) & $\mathrm{R}$ & $\mathrm{E}$ & 0 & 0 & 0 & 0 & 0 & 0 & 2 & 0 & 0 & 0 & 0 & 0 & 2 & $0,17 \pm 0,58$ & 2 & 0 & Ac \\
\hline Nettapus auritus (Boddaert, 1783) & $\mathrm{R}$ & $\bar{E}$ & 0 & 0 & 0 & 0 & 2 & 2 & 2 & 0 & 0 & 0 & 4 & 6 & 6 & $1,33 \pm 1,97$ & 16 & 0,01 & Ac \\
\hline
\end{tabular}




\begin{tabular}{|c|c|c|c|c|c|c|c|c|c|c|c|c|c|c|c|c|c|c|c|}
\hline ORDRES /Familles/Espèces & Bio & $\mathrm{Hab}$ & Jan & Fév & Mars & Avr & Mai & Juin & Juil & Août & Sept & Oct & Nov & Déc & IPA & $\begin{array}{c}\text { Moy } \\
\text { Men士Ec-Ty }\end{array}$ & EC & $\mathrm{Fr}$ & IA \\
\hline $\begin{array}{l}\text { FALCONIFORMES } \\
\text { Pandionidae } \\
\text { Pandion haliaetus (Linné, 1758) } \\
\text { Accipitridae } \\
\text { Aviceda cuculoides Swainson, } 1837\end{array}$ & $\mathrm{R}$ & $\mathrm{F}$ & & & & & & & & & & & & & & & & & \\
\hline Elanus caeruleus (Desfontaines, 1789) & $\mathrm{R}$ & f. & 6 & 5 & 3 & 2 & 5 & 4 & 4 & 4 & 5 & 4 & 4 & 6 & 6 & $4,33 \pm 1,15$ & 52 & 0,03 & Ac \\
\hline Milvus migrans (Boddaert, 1783) & $\mathrm{M} / \mathrm{P}$ & f. & 54 & 73 & 81 & 124 & 82 & 7 & 0 & 0 & 39 & 40 & 33 & 46 & 124 & $48,25 \pm 37,35$ & 579 & 0,33 & $\mathrm{Ra}$ \\
\hline Gypohierax angolensis (J. F. Gmelin, 1788) & $\mathrm{R}$ & $\mathrm{F}$ & 6 & 4 & 4 & 2 & 6 & 7 & 4 & 2 & 3 & 3 & 4 & 4 & 7 & $4,08 \pm 1,56$ & 49 & 0,03 & Ac \\
\hline $\begin{array}{l}\text { Circaetus beaudouini Verreaux \& Des Murs, } \\
1862\end{array}$ & $\mathrm{R}$ & f. & 1 & 0 & 0 & 0 & 0 & 0 & 0 & 0 & 0 & 1 & 0 & 0 & 1 & $0,17 \pm 0,39$ & 2 & 0 & Ac \\
\hline Polyboroides typus Smith, 1829 & $\mathrm{R}$ & $\mathrm{F}$ & 7 & 4 & 3 & 4 & 4 & 6 & 7 & 6 & 5 & 5 & 5 & 6 & 7 & $5,17 \pm 1,27$ & 62 & 0,04 & Ac \\
\hline Circus pygargus (Linné, 1758) & $P$ & f. & 1 & 0 & 0 & 0 & 0 & 0 & 0 & 0 & 0 & 0 & 0 & 1 & 1 & $0,17 \pm 0,39$ & 2 & 0 & Ac \\
\hline Circus aeruginosus (Linné, 1758) & $\mathrm{P}$ & f. & 2 & 6 & 3 & 0 & 0 & 0 & 0 & 0 & 0 & 1 & 2 & 3 & 6 & $1,42 \pm 1,88$ & 17 & 0,01 & Ac \\
\hline Accipiter tachiro (Daudin, 1800) & $\mathrm{R}$ & $\mathrm{F}$ & 1 & 1 & 2 & 4 & 1 & 1 & 1 & 0 & 1 & 3 & 1 & 3 & 4 & $1,58 \pm 1,16$ & 19 & 0,01 & Ac \\
\hline Accipiter badius (J. F. Gmelin, 1788) & $\mathrm{R} / \mathrm{M}$ & f. & 1 & 3 & 2 & 3 & 2 & 0 & 1 & 1 & 4 & 4 & 3 & 3 & 4 & $2,25 \pm 1,29$ & 27 & 0,02 & Ac \\
\hline Accipiter ovampensis Gurney, 1875 & $\mathrm{M}$ & $\mathrm{F}$ & 1 & 0 & 1 & 1 & 0 & 0 & 0 & 1 & 0 & 1 & 0 & 1 & 1 & $0,5 \pm 0,52$ & 6 & 0 & Ac \\
\hline Accipiter melanoleucus Smith, 1830 & $\mathrm{R}$ & $\mathrm{FF}$ & 0 & 0 & 2 & 0 & 0 & 0 & 1 & 0 & 2 & 0 & 0 & 0 & 2 & $0,42 \pm 0,79$ & 5 & 0 & Ac \\
\hline $\begin{array}{l}\text { Buteo auguralis Salvadori, } 1865 \\
\text { Falconidae }\end{array}$ & $\mathrm{R} / \mathrm{M}$ & $\mathrm{F}$ & & & & & & & & & & & & & & & & & \\
\hline Falco tinnunculus Linné, 1758 & $\mathrm{P}$ & f. & 0 & 0 & 1 & 0 & 0 & 0 & 0 & 0 & 0 & 0 & 0 & 1 & 1 & $0,17 \pm 0,39$ & 2 & 0 & Ac \\
\hline Falco ardosiaceus Vieillot, 1823 & $\mathrm{R}$ & f. & 3 & 3 & 3 & 3 & 3 & 2 & 3 & 2 & 3 & 4 & 6 & 4 & 6 & $3,25 \pm 1,06$ & 39 & 0,02 & Ac \\
\hline Falco cuvierii A. Smith, 1830 & $\mathrm{R}$ & f. & 1 & 0 & 0 & 0 & 0 & 0 & 0 & 1 & 0 & 0 & 1 & 0 & 1 & $0,25 \pm 0,45$ & 3 & 0 & Ac \\
\hline $\begin{array}{l}\text { Falco peregrinus TunStall, } 1771 \\
\text { GALLIFORMES } \\
\text { Phasianidae }\end{array}$ & $P$ & f. & 1 & 1 & 1 & 1 & 1 & 0 & 0 & 0 & 0 & 1 & 2 & 1 & 2 & $0,75 \pm 0,62$ & 9 & 0,01 & Ac \\
\hline $\begin{array}{l}\text { Coturnix adansonii J. Verreaux \& E. Verreaux, } \\
1851\end{array}$ & $\mathrm{R} / \mathrm{M}$ & f. & 0 & 0 & 0 & 0 & 0 & 0 & 0 & 0 & 0 & 1 & 0 & 0 & 1 & $0,08 \pm 0,29$ & 1 & 0 & Ac \\
\hline Francolinus ahantensis Temminck, 1854 A05 & $\mathrm{R}$ & $\mathrm{F}$ & 0 & 0 & 0 & 0 & 0 & 0 & 0 & 0 & 0 & 1 & 2 & 2 & 2 & $0,42 \pm 0,79$ & 5 & 0 & Ac \\
\hline Francolinus bicalcaratus (Linné, 1766) & $\mathrm{R}$ & f. & 2 & 3 & 1 & 2 & 2 & 2 & 5 & 7 & 4 & 2 & 4 & 5 & 7 & $3,25 \pm 1,76$ & 39 & 0,02 & Ac \\
\hline $\begin{array}{l}\text { GRUIFORMES } \\
\text { Rallidae }\end{array}$ & & & & & & & & & & & & & & & & & & & \\
\hline Sarothrura pulchra (J. E. Gray, 1829) A05 & $\mathrm{R}$ & $\mathrm{F}$ & 6 & 3 & 2 & 2 & 8 & 7 & 2 & 3 & 4 & 4 & 7 & 7 & 8 & $4,58 \pm 2,27$ & 55 & 0,03 & Ac \\
\hline Crex egregia (Peters, 1854) & $\mathrm{R} / \mathrm{M}$ & f. & 10 & 6 & 4 & 6 & 5 & 2 & 4 & 2 & 2 & 2 & 3 & 7 & 10 & $4,42 \pm 2,5$ & 53 & 0,03 & Ac \\
\hline Amaurornis flavirostra (Swainson, 1837) & $\mathrm{R}$ & $E$ & 24 & 21 & 23 & 19 & 30 & 24 & 37 & 26 & 27 & 39 & 33 & 28 & 39 & $27,58 \pm 6,19$ & 331 & 0,19 & Ac \\
\hline $\begin{array}{l}\text { Porphyrio alleni Thomson, } 1842 \\
\text { Gallinula chloropus (Linné, 1758) }\end{array}$ & $\begin{array}{c}\mathrm{R} / \mathrm{M} \\
\mathrm{R}\end{array}$ & $\begin{array}{l}\mathrm{E} \\
\mathrm{E}\end{array}$ & 1 & 4 & 3 & 1 & 22 & 43 & 34 & 24 & 6 & 3 & 5 & 5 & 43 & $12,58 \pm 14,42$ & 151 & 0,09 & Ac \\
\hline
\end{tabular}




\begin{tabular}{|c|c|c|c|c|c|c|c|c|c|c|c|c|c|c|c|c|c|c|c|}
\hline ORDRES /Familles/Espèces & Bio & $\mathrm{Hab}$ & Jan & Fév & Mars & Avr & Mai & Juin & Juil & Août & Sept & Oct & Nov & Déc & IPA & $\begin{array}{c}\text { Moy } \\
\text { Men士Ec-Ty }\end{array}$ & EC & $\mathrm{Fr}$ & IA \\
\hline $\begin{array}{l}\text { Gallinula angulata Sundevall, } 1850 \\
\text { Gruidae }\end{array}$ & $\mathrm{R} / \mathrm{M}$ & $E$ & 0 & 0 & 0 & 0 & 13 & 44 & 4 & 4 & 0 & 1 & 0 & 2 & 44 & $5,67 \pm 12,64$ & 68 & 0,04 & Ac \\
\hline $\begin{array}{l}\text { Balearica pavonina (Linné, 1758) NT } \\
\text { CHARADRIIFORMES } \\
\text { Jacanidae }\end{array}$ & 0 & f. & 0 & 0 & 0 & 0 & 0 & 0 & 0 & 2 & 0 & 0 & 0 & 0 & 2 & $0,17 \pm 0,58$ & 2 & 0 & Ac \\
\hline $\begin{array}{l}\text { Actophilornis africanus (J. F. Gmelin, 1789) } \\
\text { Rostratulidae }\end{array}$ & $\mathrm{R}$ & $E$ & 14 & 10 & 9 & 66 & 370 & 154 & 56 & 60 & 43 & 91 & 136 & 125 & 370 & $94,5 \pm 99,62$ & 1134 & 0,65 & $\mathrm{Ra}$ \\
\hline $\begin{array}{l}\text { Rostratula benghalensis (Linné, 1758) } \\
\text { Glareolidae }\end{array}$ & $\mathrm{R} / \mathrm{M}$ & $E$ & 11 & 2 & 4 & 0 & 14 & 7 & 6 & 4 & 6 & 13 & 12 & 8 & 14 & $7,25 \pm 4,47$ & 87 & 0,05 & Ac \\
\hline $\begin{array}{l}\text { Glareola nordmanni Fischer, } 1842 \\
\text { Charadriidae }\end{array}$ & 0 & f. & 0 & 0 & 1 & 0 & 0 & 0 & 0 & 0 & 0 & 0 & 0 & 0 & 1 & $0,08 \pm 0,29$ & 1 & 0 & Ac \\
\hline Charadrius hiaticula Linné, 1758 & $P$ & $E$ & 7 & 0 & 10 & 22 & 0 & 0 & 0 & 0 & 9 & 8 & 51 & 8 & 51 & $9,58 \pm 14,59$ & 115 & 0,07 & Ac \\
\hline Charadrius forbesi (Shelley, 1883) & $\mathrm{R} / \mathrm{M}$ & $E$ & 8 & 0 & 2 & 6 & 0 & 0 & 0 & 0 & 0 & 0 & 0 & 0 & 8 & $1,33 \pm 2,74$ & 16 & 0,01 & Ac \\
\hline Pluvialis dominica (Muller, 1776) & 0 & $E$ & 0 & 0 & 0 & 0 & 0 & 0 & 0 & 0 & 0 & 2 & 0 & 0 & 2 & $0,17 \pm 0,58$ & 2 & 0 & Ac \\
\hline Vanellus senegallus (Linné, 1766) & $\mathrm{R} / \mathrm{M}$ & $E$ & 0 & 0 & 0 & 0 & 1 & 0 & 0 & 0 & 0 & 0 & 0 & 0 & 1 & $0,08 \pm 0,29$ & 1 & 0 & Ac \\
\hline $\begin{array}{l}\text { Vanellus spinosus (Linné, 1758) } \\
\text { Vanellus lugubris (Lesson, 1826) } \\
\text { Scolopacidae }\end{array}$ & $\begin{array}{l}P \\
M\end{array}$ & $\begin{array}{l}E \\
f .\end{array}$ & 0 & 0 & 2 & 2 & 32 & 13 & 3 & 2 & 0 & 2 & 1 & 1 & 32 & $4,83 \pm 9,24$ & 58 & 0,03 & Ac \\
\hline Calidris canutus (Linné, 1758) & $P$ & $E$ & 0 & 0 & 0 & 0 & 0 & 0 & 0 & 0 & 0 & 1 & 0 & 0 & 1 & $0,08 \pm 0,29$ & 1 & 0 & Ac \\
\hline Calidris minuta (Leisler, 1812) & $P$ & $\mathrm{E}$ & 0 & 0 & 0 & 0 & 0 & 0 & 0 & 0 & 0 & 2 & 1 & 0 & 2 & $0,25 \pm 0,62$ & 3 & 0 & Ac \\
\hline Calidris fuscicollis (Vieillot, 1819) & 0 & $E$ & 0 & 0 & 0 & 0 & 0 & 0 & 0 & 0 & 0 & 1 & 0 & 0 & 1 & $0,08 \pm 0,29$ & 1 & 0 & Ac \\
\hline Calidris melanotos (Vieillot, 1819) & 0 & E & 0 & 0 & 0 & 0 & 0 & 0 & 0 & 0 & 0 & 1 & 1 & 0 & 1 & $0,17 \pm 0,39$ & 2 & 0 & Ac \\
\hline Calidris ferruginea (Pontoppidan, 1763) & $P$ & $E$ & 0 & 0 & 0 & 0 & 0 & 0 & 0 & 0 & 3 & 11 & 0 & 0 & 11 & $1,17 \pm 3,21$ & 14 & 0,01 & Ac \\
\hline Gallinago gallinago (Linné, 1758) & $P$ & $\mathrm{E}$ & 17 & 2 & 1 & 0 & 0 & 0 & 0 & 0 & 0 & 1 & 8 & 30 & 30 & $4,92 \pm 9,39$ & 59 & 0,03 & Ac \\
\hline Numenius phaeopus (Linné, 1758) & $P$ & $\bar{E}$ & 1 & 3 & 1 & 0 & 0 & 0 & 0 & 7 & 7 & 0 & 1 & 0 & 7 & $1,67 \pm 2,64$ & 20 & 0,01 & Ac \\
\hline Tringa totanus (Linné, 1758) & $P$ & $E$ & 0 & 0 & 0 & 0 & 0 & 0 & 0 & 0 & 0 & 0 & 0 & 1 & 1 & $0,08 \pm 0,29$ & 1 & 0 & Ac \\
\hline Tringa stagnatilis (Bechstein, 1803) & $P$ & $E$ & 2 & 0 & 0 & 0 & 0 & 0 & 0 & 0 & 0 & 0 & 1 & 1 & 2 & $0,33 \pm 0,65$ & 4 & 0 & Ac \\
\hline Tringa nebularia (Gunnerus, 1767) & $P$ & $E$ & 2 & 0 & 1 & 0 & 2 & 0 & 0 & 2 & 2 & 3 & 6 & 5 & 6 & $1,92 \pm 1,98$ & 23 & 0,01 & Ac \\
\hline Tringa ochropus Linné, 1758 & $P$ & $E$ & 1 & 0 & 0 & 0 & 0 & 0 & 0 & 0 & 0 & 0 & 0 & 2 & 2 & $0,25 \pm 0,62$ & 3 & 0 & Ac \\
\hline Tringa glareola (Linné, 1758) & $P$ & $E$ & 15 & 32 & 36 & 31 & 1 & 0 & 18 & 24 & 64 & 56 & 90 & 45 & 90 & $34,33 \pm 26,32$ & 412 & 0,24 & $\mathrm{Ra}$ \\
\hline $\begin{array}{l}\text { Actitis hypoleucos (Linné, 1758) } \\
\text { COLUMBIFORMES } \\
\text { Columbidae }\end{array}$ & $P$ & $E$ & 5 & 0 & 11 & 3 & 1 & 0 & 0 & 4 & 10 & 16 & 11 & 7 & 16 & $5,67 \pm 5,33$ & 68 & 0,04 & Ac \\
\hline Treron calvus (Temminck, 1808) & $\mathrm{R}$ & $\mathrm{F}$ & 53 & 51 & 48 & 21 & 22 & 61 & 28 & 22 & 49 & 36 & 11 & 27 & 61 & $35,75 \pm 16,06$ & 429 & 0,25 & $\mathrm{Ra}$ \\
\hline Turtur tympanistria (Temminck, 1809) & $\mathrm{R}$ & $\mathrm{F}$ & 21 & 18 & 14 & 6 & 2 & 6 & 11 & 15 & 18 & 23 & 17 & 16 & 23 & $13,92 \pm 6,44$ & 167 & 0,1 & Ac \\
\hline Turtur afer (Linné, 1766) & $\mathrm{R}$ & f. & 59 & 54 & 43 & 27 & 43 & 46 & 59 & 61 & 80 & 71 & 60 & 63 & 80 & $55,5 \pm 14,11$ & 666 & 0,38 & $\mathrm{Ra}$ \\
\hline
\end{tabular}




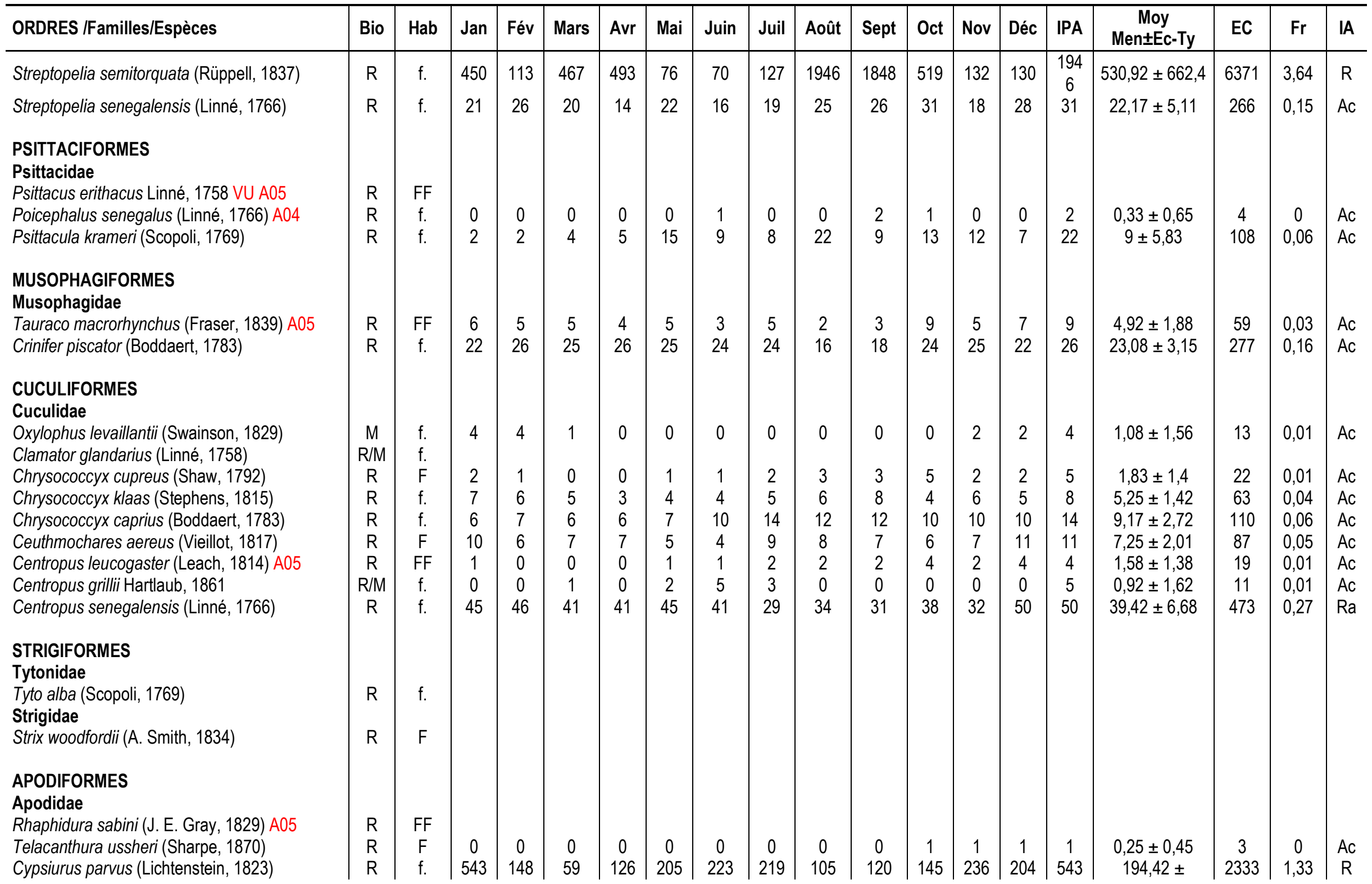




\begin{tabular}{|c|c|c|c|c|c|c|c|c|c|c|c|c|c|c|c|c|c|c|c|}
\hline ORDRES /Familles/Espèces & Bio & $\mathrm{Hab}$ & Jan & Fév & Mars & Avr & Mai & Juin & Juil & Août & Sept & Oct & Nov & Déc & IPA & $\begin{array}{c}\text { Moy } \\
\text { Men士Ec-Ty }\end{array}$ & EC & $\mathrm{Fr}$ & IA \\
\hline Anus anus (Linné 1758) & $P$ & $f$ & 54 & 0 & 92 & 0 & 8 & 0 & 0 & 0 & 3 & 0 & 2 & 1 & 92 & $\begin{array}{c}122,85 \\
1333+2912\end{array}$ & 160 & 009 & $A c$ \\
\hline Apus caffer (Lichtenstein, 1823) & $\mathrm{R} / \mathrm{M}$ & f. & 0 & 0 & 0 & 0 & 5 & 1 & 1 & 0 & 0 & 0 & 0 & 0 & 5 & $0,58 \pm 1,44$ & 7 & 0 & $\mathrm{Ac}$ \\
\hline Apus affinis (J. E. Gray, 1830) & $\mathrm{R}$ & f. & 409 & 254 & 130 & 130 & 165 & 148 & 196 & 206 & 232 & 160 & 214 & 173 & 409 & $201,42 \pm 76,22$ & 2417 & 1,38 & $\mathrm{R}$ \\
\hline \multicolumn{20}{|l|}{$\begin{array}{l}\text { CORACIIFORMES } \\
\text { Alcedinidae }\end{array}$} \\
\hline Halcyon malimbica (Shaw, 1811) & $\mathrm{R}$ & $\mathrm{F}$ & 3 & 3 & 6 & 5 & 4 & 3 & 7 & 5 & 4 & 5 & 3 & 3 & 7 & $4,25 \pm 1,36$ & 51 & 0,03 & $A c$ \\
\hline Halcyon senegalensis (Linné, 1766) & $\mathrm{R}$ & f. & 16 & 15 & 16 & 19 & 27 & 18 & 15 & 12 & 7 & 14 & 17 & 15 & 27 & $15,92 \pm 4,66$ & 191 & 0,11 & Ac \\
\hline Ceyx pictus (Boddaert, 1783) & $\mathrm{R} / \mathrm{M}$ & f. & 1 & 3 & 1 & 4 & 4 & 0 & 1 & 2 & 2 & 0 & 3 & 2 & 4 & $1,92 \pm 1,38$ & 23 & 0,01 & $A c$ \\
\hline Alcedo cristata Pallas, 1764 & $\mathrm{R}$ & $\mathrm{E}$ & 1 & 2 & 3 & 4 & 7 & 6 & 1 & 3 & 7 & 4 & 5 & 6 & 7 & $4,08 \pm 2,15$ & 49 & 0,03 & $A c$ \\
\hline $\begin{array}{l}\text { Ceryle rudis (Linné, 1758) } \\
\text { Meropidae }\end{array}$ & $\mathrm{R}$ & $\mathrm{E}$ & 1 & 0 & 1 & 0 & 2 & 3 & 1 & 1 & 1 & 6 & 6 & 2 & 6 & $2 \pm 2,04$ & 24 & 0,01 & $A c$ \\
\hline Merops gularis Shaw, 1798 A05 & $\mathrm{R}$ & $\mathrm{FF}$ & 3 & 2 & 1 & 0 & 0 & 0 & 0 & 0 & 0 & 2 & 2 & 3 & 3 & $1,08 \pm 1,24$ & 13 & 0,01 & $A c$ \\
\hline Merops pusillus Muller, 1776 & $\mathrm{R}$ & f. & 34 & 33 & 32 & 43 & 43 & 32 & 23 & 41 & 26 & 34 & 46 & 41 & 46 & $35,67 \pm 7,16$ & 428 & 0,24 & $\mathrm{Ra}$ \\
\hline $\begin{array}{l}\text { Merops albicollis Vieillot, } 1817 \\
\text { Coraciidae }\end{array}$ & M & f. & 60 & 200 & 80 & 104 & 173 & 0 & 0 & 0 & 0 & 0 & 82 & 265 & 265 & $80,33 \pm 90,58$ & 964 & 0,55 & $\mathrm{Ra}$ \\
\hline $\begin{array}{l}\text { Eurystomus glaucurus (Statius Muller, 1776) } \\
\text { Bucerotidae }\end{array}$ & M & f. & 106 & 41 & 4 & 0 & 0 & 0 & 0 & 0 & 0 & 0 & 3 & 51 & 106 & $17,08 \pm 33,09$ & 205 & 0,12 & Ac \\
\hline Tropicranus albocristatus (Cassin, 1848) A05 & $\mathrm{R}$ & FF & & & & & & & & & & & & & & & & & \\
\hline Tockus fasciatus (Shaw, 1811) A05 & $\mathrm{R}$ & $\mathrm{F}$ & 53 & 34 & 33 & 25 & 26 & 32 & 36 & 70 & 60 & 54 & 58 & 59 & 70 & $45 \pm 15,48$ & 540 & 0,31 & $\mathrm{Ra}$ \\
\hline Bycanistes fistulator (Cassin, 1850) A05 & $\mathrm{R}$ & $\mathrm{FF}$ & 31 & 22 & 34 & 33 & 13 & 32 & 61 & 42 & 31 & 20 & 11 & 20 & 61 & $29,17 \pm 13,62$ & 350 & 0,2 & $\mathrm{Ra}$ \\
\hline \multicolumn{20}{|l|}{$\begin{array}{l}\text { PICIFORMES } \\
\text { Capitonidae }\end{array}$} \\
\hline Gymnobucco calvus (Lafresnaye, 1841) A05 & $\mathrm{R}$ & $\mathrm{F}$ & 6 & 2 & 0 & 0 & 0 & 2 & 0 & 2 & 2 & 0 & 7 & 5 & 7 & $2,17 \pm 2,52$ & 26 & 0,01 & Ac \\
\hline Pogoniulus scolopaceus (Bonaparte, 1850) A05 & $\mathrm{R}$ & $\mathrm{F}$ & 4 & 4 & 3 & 4 & 4 & 4 & 5 & 4 & 5 & 5 & 4 & 5 & 5 & $4,25 \pm 0,62$ & 51 & 0,03 & Ac \\
\hline Pogoniulus atroflavus (Sparrman, 1798) A05 & $\mathrm{R}$ & $\mathrm{FF}$ & 1 & 0 & 1 & 0 & 1 & 1 & 4 & 2 & 2 & 5 & 3 & 3 & 5 & $1,92 \pm 1,56$ & 23 & 0,01 & $A c$ \\
\hline Pogoniulus subsulphureus (Fraser, 1843) A05 & $\mathrm{R}$ & $\mathrm{FF}$ & 5 & 4 & 4 & 3 & 1 & 3 & 5 & 5 & 6 & 4 & 7 & 5 & 7 & $4,33 \pm 1,56$ & 52 & 0,03 & Ac \\
\hline Pogoniulus bilineatus (Sundevall, 1850) & $\mathrm{R}$ & $\mathrm{F}$ & 11 & 13 & 10 & 10 & 6 & 9 & 7 & 6 & 13 & 9 & 10 & 6 & 13 & $9,17 \pm 2,52$ & 110 & 0,06 & $\mathrm{Ac}$ \\
\hline Tricholaema hirsuta (Swainson, 1821) A05 & $\mathrm{R}$ & $\mathrm{F}$ & 0 & 1 & 2 & 2 & 0 & 1 & 1 & 1 & 0 & 1 & 1 & 1 & 2 & $0,92 \pm 0,67$ & 11 & 0,01 & Ac \\
\hline Lybius vieilloti (Leach, 1815) & $\mathrm{R}$ & f. & 10 & 6 & 8 & 12 & 6 & 7 & 8 & 6 & 6 & 6 & 8 & 5 & 12 & $7,33 \pm 2,02$ & 88 & 0,05 & Ac \\
\hline Lybius bidentatus (Shaw, 1799) & $\mathrm{R}$ & f. & 2 & 1 & 1 & 2 & 0 & 2 & 0 & 0 & 1 & 0 & 0 & 1 & 2 & $0,83 \pm 0,83$ & 10 & 0,01 & Ac \\
\hline Lybius dubius (Gmelin, 1788) A04 & $\mathrm{R}$ & f. & 0 & 1 & 0 & 0 & 0 & 0 & 0 & 0 & 0 & 0 & 0 & 0 & 1 & $0,08 \pm 0,29$ & 1 & 0 & Ac \\
\hline $\begin{array}{l}\text { Trachylaemus purpuratus (J. Verreaux \& E. } \\
\text { Verreaux, 1851) A05 }\end{array}$ & $\mathrm{R}$ & $\mathrm{F}$ & & & & & & & & & & & & & & & & & \\
\hline Indicatoridae & & & & & & & & & & & & & & & & & & & \\
\hline Indicator conirostris (Cassin, 1856) & $\mathrm{R}$ & $\mathrm{F}$ & & & & & & & & & & & & & & & & & \\
\hline
\end{tabular}




\begin{tabular}{|c|c|c|c|c|c|c|c|c|c|c|c|c|c|c|c|c|c|c|c|}
\hline ORDRES /Familles/Espèces & Bio & Hab & Jan & Fév & Mars & Avr & Mai & Juin & Juil & Août & Sept & Oct & Nov & Déc & IPA & $\begin{array}{c}\text { Moy } \\
\text { Men士Ec-Ty }\end{array}$ & EC & $\mathrm{Fr}$ & IA \\
\hline \multicolumn{20}{|l|}{ Picidae } \\
\hline Campethera nivosa & $\mathrm{R}$ & f. & 2 & 0 & 1 & 5 & 4 & 2 & 4 & 3 & 7 & 3 & 4 & 2 & 7 & $3,08 \pm 1,88$ & 37 & 0,02 & $A c$ \\
\hline $\begin{array}{l}\text { Dendropicos gabonensis (J. Verreaux \& E. } \\
\text { Verreaux, 1851) A05 }\end{array}$ & $\mathrm{R}$ & f. & 1 & 0 & 0 & 0 & 0 & 0 & 0 & 0 & 0 & 3 & 0 & 0 & 3 & $0,33 \pm 0,89$ & 4 & 0 & Ac \\
\hline $\begin{array}{l}\text { Dendropicos pyrrhogaster (Malherbe, 1845) } \\
\text { A05 }\end{array}$ & $\mathrm{R}$ & $\mathrm{F}$ & 0 & 0 & 1 & 0 & 2 & 2 & 0 & 0 & 0 & 1 & 2 & 2 & 2 & $0,83 \pm 0,94$ & 10 & 0,01 & $A c$ \\
\hline Dendropicos goertae (Statius Muller, 1776) & $\mathrm{R}$ & f. & 2 & 2 & 2 & 3 & 1 & 2 & 0 & 1 & 1 & 1 & 2 & 0 & 3 & $1,42 \pm 0,9$ & 17 & 0,01 & Ac \\
\hline \multicolumn{20}{|l|}{ PASSERIFORMES } \\
\hline Psalidoprocne obscura (Hartlaub, 1855) A05 & $\mathrm{R} / \mathrm{M}$ & $\mathrm{F}$ & 16 & 50 & 25 & 10 & 13 & 9 & 1 & 0 & 1 & 14 & 27 & 9 & 50 & $14,58 \pm 14,1$ & 175 & 0,1 & 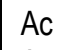 \\
\hline Riparia riparia (Linné, 1758) & $P$ & f. & 3 & 2 & 5 & 0 & 0 & 0 & 0 & 0 & 0 & 0 & 6 & 2 & 6 & $1,5 \pm 2,15$ & 18 & 0,01 & Ac \\
\hline Hirundo semirufa Sundevall, 1850 & $\mathrm{R}$ & f. & 54 & 44 & 30 & 25 & 21 & 14 & 18 & 17 & 38 & 11 & 15 & 12 & 54 & $24,92 \pm 13,85$ & 299 & 0,17 & Ac \\
\hline Hirundo abyssinica Guerin-Meneville, 1843 & $\mathrm{R} / \mathrm{M}$ & f. & 85 & 81 & 36 & 40 & 28 & 26 & 39 & 23 & 41 & 57 & 79 & 117 & 117 & $54,33 \pm 29,58$ & 652 & 0,37 & $\mathrm{Ra}$ \\
\hline Hirundo nigrita Gray, 1845A05 & $\mathrm{R}$ & $E$ & 1 & 0 & 0 & 0 & 1 & 0 & 1 & 1 & 0 & 2 & 3 & 1 & 3 & $0,83 \pm 0,94$ & 10 & 0,01 & Ac \\
\hline Hirundo aethiopica Blanford, 1869 & $\mathrm{R} / \mathrm{M}$ & f. & 13 & 25 & 15 & 7 & 33 & 11 & 7 & 10 & 10 & 13 & 18 & 18 & 33 & $15 \pm 7,65$ & 180 & 0,1 & Ac \\
\hline Hirundo rustica Linné, 1758 & $P$ & f. & 443 & $\begin{array}{c}620 \\
6\end{array}$ & 77 & 28 & 0 & 0 & 0 & 0 & 0 & 50 & 153 & 252 & $\begin{array}{c}620 \\
6\end{array}$ & $\begin{array}{c}600,75 \pm \\
1770,36\end{array}$ & 7209 & 4,12 & R \\
\hline \multicolumn{20}{|l|}{ Motacillidae } \\
\hline Motacilla flava Linné, 1758 & $P$ & f. & 34 & 181 & 97 & 107 & 0 & 0 & 0 & 0 & 0 & 9 & 240 & 35 & 240 & $58,58 \pm 81,18$ & 703 & 0,4 & $\mathrm{Ra}$ \\
\hline Motacilla aguimp Dumont, 1 & $\mathrm{R}$ & f. & & & & & & & & & & & & & & & & & \\
\hline Anthus leucophrys Vieillot, 1818 & $\mathrm{R}$ & f. & 12 & 16 & 17 & 21 & 17 & 9 & 10 & 13 & 12 & 8 & 17 & 11 & 21 & $13,58 \pm 3,96$ & 163 & 0,09 & Ac \\
\hline Macronyx croceus (Vieillot, 1816) & $\mathrm{R}$ & f. & 13 & 12 & 11 & 11 & 11 & 9 & 11 & 9 & 7 & 6 & 9 & 10 & 13 & $9,92 \pm 2,02$ & 119 & 0,07 & Ac \\
\hline \multicolumn{20}{|l|}{ Pycnonotidae } \\
\hline Andropadus virens Cassin, 1858 & $\mathrm{R}$ & $\mathrm{F}$ & 46 & 45 & 38 & 36 & 37 & 50 & 47 & 66 & 59 & 60 & 60 & 51 & 66 & $49,58 \pm 9,98$ & 595 & 0,34 & $\mathrm{Ra}$ \\
\hline Andropadus gracilis Cabanis, 1880 A05 & $\mathrm{R}$ & $\mathrm{F}$ & 0 & 3 & 2 & 0 & 0 & 1 & 1 & 1 & 2 & 1 & 2 & 3 & 3 & $1,33 \pm 1,07$ & 16 & 0,01 & Ac \\
\hline Andropadus curvirostris Cassin, 1859 A05 & $\mathrm{R}$ & $\mathrm{FF}$ & 1 & 0 & 4 & 1 & 2 & 1 & 2 & 3 & 1 & 2 & 2 & 2 & 4 & $1,75 \pm 1,06$ & 21 & 0,01 & Ac \\
\hline ilirostris Strickland, 1844 & $\mathrm{R}$ & $\mathrm{FF}$ & 3 & 1 & 2 & 1 & 3 & 1 & 1 & 2 & 5 & 3 & 3 & 2 & 5 & $2,25 \pm 1,22$ & 27 & 0,02 & Ac \\
\hline Andropadus latirostris Strickland, 1844 & $\mathrm{R}$ & $\mathrm{F}$ & 2 & 0 & 0 & 0 & 0 & 2 & 1 & 2 & 2 & 2 & 2 & 2 & 2 & $1,25 \pm 0,97$ & 15 & 0,01 & Ac \\
\hline $\begin{array}{l}\text { Baeopogon indicator (Verreaux \& Verreaux, } \\
\text { 1855) A05 }\end{array}$ & $\mathrm{R}$ & $\mathrm{F}$ & & & & & & & & & & & & & & & & & \\
\hline Chlorocichla simplex (Hartlaub, 1855) A05 & $\mathrm{R}$ & $\mathrm{F}$ & 29 & 20 & 15 & 15 & 15 & 17 & 25 & 23 & 25 & 33 & 24 & 27 & 33 & $22,33 \pm 5,97$ & 268 & 0,15 & Ac \\
\hline Thescelocichla leucopleura (Cassin, 1855) A05 & $\mathrm{R}$ & $\mathrm{F}$ & 16 & 11 & 14 & 11 & 10 & 13 & 18 & 17 & 16 & 19 & 17 & 23 & 23 & $15,42 \pm 3,8$ & 185 & 0,11 & Ac \\
\hline Bleda canicapillus (Hartlaub, 1854) A05 & $\mathrm{R}$ & FF & 2 & 2 & 3 & 0 & 0 & 0 & 3 & 1 & 2 & 4 & 3 & 2 & 4 & $1,83 \pm 1,34$ & 22 & 0,01 & Ac \\
\hline Criniger calurus (Cassin, 1857) A05 & $\mathrm{R}$ & FF & 3 & 0 & 2 & 2 & 2 & 0 & 1 & 2 & 3 & 1 & 2 & 2 & 3 & $1,67 \pm 0,98$ & 20 & 0,01 & Ac \\
\hline Pycnonotus barbatus (Desfontaines, 1789) & $\mathrm{R}$ & $\mathrm{f}$. & 71 & 71 & 63 & 56 & 68 & 44 & 65 & 61 & 64 & 136 & 81 & 63 & 136 & $70,25 \pm 22,55$ & 843 & 0,48 & $\mathrm{Ra}$ \\
\hline $\begin{array}{l}\text { Nicator chloris (Valenciennes, 1826) A05 } \\
\text { Turdidae }\end{array}$ & $\mathrm{R}$ & $F$ & 2 & 4 & 4 & 4 & 3 & 3 & 1 & 4 & 4 & 4 & 3 & 3 & 4 & $3,25 \pm 0,97$ & 39 & 0,02 & Ac \\
\hline
\end{tabular}




\begin{tabular}{|c|c|c|c|c|c|c|c|c|c|c|c|c|c|c|c|c|c|c|c|}
\hline ORDRES /Familles/Espèces & Bio & $\mathrm{Hab}$ & Jan & Fév & Mars & Avr & Mai & Juin & Juil & Août & Sept & Oct & Nov & Déc & IPA & $\begin{array}{c}\text { Moy } \\
\text { Men士Ec-Ty }\end{array}$ & EC & $\mathrm{Fr}$ & IA \\
\hline Cossypha niveicapilla (Lafresnaye, 1838) & $\mathrm{R}$ & $\mathrm{F}$ & 2 & 2 & 2 & 3 & 6 & 3 & 4 & 2 & 3 & 3 & 4 & 3 & 6 & $3,08 \pm 1,16$ & 37 & 0,02 & Ac \\
\hline Saxicola rubetra (Linné, 1758) & $P$ & f. & 1 & 3 & 6 & 0 & 0 & 0 & 0 & 0 & 0 & 0 & 0 & 1 & 6 & $0,92 \pm 1,83$ & 11 & 0,01 & Ac \\
\hline $\begin{array}{l}\text { Turdus pelios Bonaparte, } 1850 \\
\text { Sylviidae }\end{array}$ & $\mathrm{R}$ & f. & 0 & 0 & 3 & 2 & 2 & 3 & 0 & 1 & 0 & 0 & 1 & 0 & 3 & $1 \pm 1,21$ & 12 & 0,01 & Ac \\
\hline Acrocephalus schoenobaenus (Linné, 1758) & $P$ & f. & 6 & 8 & 5 & 3 & 0 & 0 & 0 & 0 & 0 & 0 & 3 & 4 & 8 & $2,42 \pm 2,84$ & 29 & 0,02 & Ac \\
\hline Acrocephalus scirpaceus (Hermann, 1804) & $P$ & f. & 8 & 15 & 11 & 0 & 0 & 0 & 0 & 0 & 0 & 0 & 2 & 5 & 15 & $3,42 \pm 5,21$ & 41 & 0,02 & Ac \\
\hline Acrocephalus arundinaceus (Linné, 1758) & $P$ & f. & 3 & 2 & 3 & 0 & 0 & 0 & 0 & 0 & 0 & 0 & 0 & 1 & 3 & $0,75 \pm 1,22$ & 9 & 0,01 & Ac \\
\hline $\begin{array}{l}\text { Acrocephalus rufescens (Sharpe \& Bouvier, } \\
\text { 1876) }\end{array}$ & $\mathrm{R}$ & f. & 0 & 0 & 0 & 0 & 2 & 1 & 2 & 1 & 0 & 1 & 0 & 1 & 2 & $0,67 \pm 0,78$ & 8 & 0 & Ac \\
\hline Cisticola erythrops (Hartlaub, 1857) & $\mathrm{R}$ & f. & 35 & 28 & 30 & 32 & 25 & 22 & 34 & 25 & 22 & 27 & 32 & 25 & 35 & $28,08 \pm 4,48$ & 337 & 0,19 & Ac \\
\hline Cisticola galactotes (Temminck, 1821) & $\mathrm{R}$ & f. & 80 & 63 & 72 & 61 & 68 & 75 & 102 & 71 & 72 & 56 & 63 & 62 & 102 & $70,42 \pm 12,06$ & 845 & 0,48 & $\mathrm{Ra}$ \\
\hline Cisticola brachypterus (Sharpe, 1870) & $\mathrm{R}$ & f. & 36 & 32 & 21 & 18 & 23 & 20 & 31 & 38 & 30 & 21 & 11 & 24 & 38 & $25,42 \pm 8,01$ & 305 & 0,17 & Ac \\
\hline Prinia subflava (J. F. Gmelin, 1789) & $\mathrm{R}$ & f. & 36 & 35 & 46 & 41 & 32 & 34 & 25 & 37 & 31 & 47 & 39 & 36 & 47 & $36,58 \pm 6,17$ & 439 & 0,25 & $\mathrm{Ra}$ \\
\hline Camaroptera brachyura (Vieillot, 1820) & $\mathrm{R}$ & f. & 20 & 28 & 30 & 27 & 23 & 19 & 24 & 25 & 32 & 26 & 27 & 16 & 32 & $24,75 \pm 4,65$ & 297 & 0,17 & Ac \\
\hline Camaroptera superciliaris (Fraser, 1843) A05 & $\mathrm{R}$ & $\mathrm{FF}$ & 7 & 4 & 8 & 4 & 6 & 4 & 4 & 4 & 5 & 6 & 8 & 6 & 8 & $5,5 \pm 1,57$ & 66 & 0,04 & $A c$ \\
\hline Camaroptera chloronota Reichenow, 1895 A05 & $\mathrm{R}$ & FF & 4 & 2 & 5 & 1 & 3 & 3 & 3 & 4 & 7 & 4 & 3 & 7 & 7 & $3,83 \pm 1,8$ & 46 & 0,03 & Ac \\
\hline Macrosphenus kempi (Sharpe, 1905) A05 & $\mathrm{R}$ & $\mathrm{F}$ & 3 & 0 & 0 & 0 & 0 & 0 & 0 & 0 & 1 & 2 & 2 & 2 & 3 & $0,83 \pm 1,11$ & 10 & 0,01 & Ac \\
\hline Macrosphenus concolor (Hartlaub, 1857) A05 & $\mathrm{R}$ & $\mathrm{FF}$ & 2 & 0 & 0 & 0 & 0 & 0 & 0 & 0 & 2 & 0 & 2 & 2 & 2 & $0,67 \pm 0,98$ & 8 & 0 & Ac \\
\hline Sylvietta virens Cassin, 1859 A05 & $\mathrm{R}$ & $\mathrm{F}$ & 13 & 13 & 16 & 15 & 14 & 12 & 15 & 18 & 15 & 14 & 20 & 19 & 20 & $15,33 \pm 2,5$ & 184 & 0,11 & Ac \\
\hline $\begin{array}{l}\text { Phylloscopus trochilus (Linné, 1758) } \\
\text { Phylloscopus sibilatrix (Bechstein, 1793) }\end{array}$ & $\begin{array}{l}P \\
P\end{array}$ & $\begin{array}{l}f . \\
F\end{array}$ & 0 & 0 & 0 & 0 & 0 & 0 & 0 & 0 & 0 & 3 & 1 & 0 & 3 & $0,33 \pm 0,89$ & 4 & 0 & Ac \\
\hline $\begin{array}{l}\text { Hylia prasina (Cassin, 1855) A05 } \\
\text { Muscicapidae }\end{array}$ & $\mathrm{R}$ & $\mathrm{F}$ & 12 & 10 & 10 & 9 & 6 & 8 & 11 & 12 & 9 & 9 & 13 & 11 & 13 & $10 \pm 1,95$ & 120 & 0,07 & Ac \\
\hline Muscicapa striata (Pallas, 1764) & $P$ & f. & 0 & 0 & 0 & 0 & 0 & 0 & 0 & 0 & 0 & 0 & 2 & 0 & 2 & $0,17 \pm 0,58$ & 2 & 0 & Ac \\
\hline $\begin{array}{l}\text { Muscicapa cassini Heine, } 1860 \text { A05 } \\
\text { Monarchidae }\end{array}$ & $\mathrm{R}$ & $\mathrm{F}$ & 1 & 4 & 2 & 0 & 4 & 0 & 1 & 0 & 1 & 0 & 2 & 3 & 4 & $1,5 \pm 1,51$ & 18 & 0,01 & Ac \\
\hline $\begin{array}{l}\text { Terpsiphone rufiventer (Swainson, 1837) A05 } \\
\text { Platysteiridae }\end{array}$ & $\mathrm{R}$ & $\mathrm{F}$ & 2 & 5 & 2 & 1 & 0 & 2 & 0 & 2 & 1 & 2 & 2 & 2 & 5 & $1,75 \pm 1,29$ & 21 & 0,01 & Ac \\
\hline Dyaphorophyia castanea Fraser, 1843 A05 & $\mathrm{R}$ & $\mathrm{FF}$ & 17 & 0 & 0 & 0 & 0 & 0 & 0 & 0 & 0 & 0 & 0 & 0 & 17 & $1,42 \pm 4,91$ & 17 & 0,01 & Ac \\
\hline Dyaphorophyia blissetti (Sharpe, 1872) A05 & $\mathrm{R}$ & $\mathrm{F}$ & 1 & 0 & 0 & 0 & 0 & 0 & 0 & 0 & 0 & 2 & 3 & 1 & 3 & $0,58 \pm 1$ & 7 & 0 & Ac \\
\hline $\begin{array}{l}\text { Platysteira cyanea (Muller, 1776) } \\
\text { Remizidae }\end{array}$ & $\mathrm{R}$ & f. & 22 & 20 & 26 & 24 & 23 & 14 & 22 & 16 & 18 & 18 & 23 & 24 & 26 & $20,83 \pm 3,64$ & 250 & 0,14 & Ac \\
\hline $\begin{array}{l}\text { Pholidornis rushiae (Cassin, 1855) A05 } \\
\text { Nectariniidae }\end{array}$ & $\mathrm{R}$ & $\mathrm{FF}$ & 1 & 2 & 1 & 2 & 0 & 2 & 2 & 0 & 0 & 3 & 0 & 0 & 3 & $1,08 \pm 1,08$ & 13 & 0,01 & Ac \\
\hline Anthreptes rectirostris (Shaw, 1812) A05 & $\mathrm{R}$ & $\mathrm{FF}$ & 0 & 2 & 0 & 0 & 0 & 0 & 0 & 0 & 1 & 0 & 0 & 0 & 2 & $0,25 \pm 0,62$ & 3 & 0 & Ac \\
\hline Anabathmis reichenbachii Hartlaub, 1857 A05 & $\mathrm{R}$ & f. & 7 & 9 & 3 & 5 & 4 & 6 & 4 & 6 & 6 & 4 & 6 & 6 & 9 & $5,5 \pm 1,62$ & 66 & 0,04 & Ac \\
\hline $\begin{array}{l}\text { Cyanomitra verticalis (Latham, 1790) } \\
\text { Cyanomitra cyanolaema (Jardine \& Fraser, }\end{array}$ & $\begin{array}{l}\mathrm{R} \\
\mathrm{R}\end{array}$ & $\begin{array}{l}\mathrm{F} \\
\mathrm{FF}\end{array}$ & 4 & 4 & 6 & 7 & 6 & 5 & 5 & 3 & 8 & 8 & 6 & 12 & 12 & $6,17 \pm 2,41$ & 74 & 0,04 & Ac \\
\hline
\end{tabular}




\begin{tabular}{|c|c|c|c|c|c|c|c|c|c|c|c|c|c|c|c|c|c|c|c|}
\hline ORDRES /Familles/Espèces & Bio & $\mathrm{Hab}$ & Jan & Fév & Mars & Avr & Mai & Juin & Juil & Août & Sept & Oct & Nov & Déc & IPA & $\begin{array}{c}\text { Moy } \\
\text { Men士Ec-Ty }\end{array}$ & EC & $\mathrm{Fr}$ & IA \\
\hline 1851) A05 & & & & & & & & & & & & & & & & & & & \\
\hline Cyanomitra olivacea (Smith, 1840) & $\mathrm{R}$ & $\mathrm{FF}$ & 3 & 1 & 0 & 2 & 2 & 1 & 2 & 2 & 0 & 2 & 2 & 1 & 3 & $1,5 \pm 0,9$ & 18 & 0,01 & Ac \\
\hline Chalcomitra adelberti (Gervais, 1834) A05 & $\mathrm{R}$ & $\mathrm{FF}$ & 3 & 3 & 3 & 2 & 6 & 4 & 3 & 6 & 3 & 1 & 5 & 5 & 6 & $3,67 \pm 1,56$ & 44 & 0,03 & Ac \\
\hline Hedydipna collaris (Vieillot, 1819) & $\mathrm{R}$ & $\mathrm{F}$ & 10 & 11 & 9 & 18 & 8 & 8 & 6 & 7 & 10 & 9 & 8 & 6 & 18 & $9,17 \pm 3,19$ & 110 & 0,06 & Ac \\
\hline Cinnyris chloropygius (Jardine, 1842) & $\mathrm{R}$ & $\mathrm{F}$ & 43 & 26 & 16 & 13 & 17 & 16 & 19 & 23 & 25 & 25 & 27 & 36 & 43 & $23,83 \pm 8,76$ & 286 & 0,16 & Ac \\
\hline Cinnyris superbus (Shaw, 1812) A05 & $\mathrm{R}$ & $\mathrm{F}$ & 1 & 1 & 1 & 0 & 3 & 0 & 0 & 0 & 1 & 2 & 2 & 1 & 3 & $1 \pm 0,95$ & 12 & 0,01 & Ac \\
\hline Cinnyris coccinigastrus (Latham, 1802)A04 & $\mathrm{R}$ & f. & 13 & 11 & 10 & 9 & 6 & 9 & 9 & 7 & 5 & 9 & 7 & 9 & 13 & $8,67 \pm 2,19$ & 104 & 0,06 & Ac \\
\hline $\begin{array}{l}\text { Cinnyris cupreus (Shaw, 1812) } \\
\text { Zosteropidae }\end{array}$ & $\mathrm{R}$ & f. & 29 & 27 & 25 & 28 & 28 & 28 & 35 & 30 & 34 & 40 & 41 & 39 & 41 & $32 \pm 5,58$ & 384 & 0,22 & $\mathrm{Ra}$ \\
\hline $\begin{array}{l}\text { Zosterops senegalensis Bonaparte, } 1850 \\
\text { Laniidae }\end{array}$ & $\mathrm{R}$ & f. & & & & & & & & & & & & & & & & & \\
\hline $\begin{array}{l}\text { Lanius collaris Linné, } 1766 \\
\text { Malaconotidae }\end{array}$ & $\mathrm{R}$ & f. & 8 & 1 & 2 & 5 & 6 & 4 & 0 & 2 & 3 & 4 & 6 & 7 & 8 & $4 \pm 2,49$ & 48 & 0,03 & Ac \\
\hline Dryoscopus gambensis (Lichtenstein, 1823) & $\mathrm{R}$ & $\mathrm{F}$ & 3 & 1 & 2 & 3 & 2 & 0 & 1 & 2 & 0 & 2 & 0 & 1 & 3 & $1,42 \pm 1,08$ & 17 & 0,01 & Ac \\
\hline $\begin{array}{l}\text { Laniarius leucorhynchus (Hartlaub, 1848) A05 } \\
\text { Corvidae }\end{array}$ & $\mathrm{R}$ & f. & 4 & 5 & 3 & 2 & 2 & 4 & 2 & 2 & 3 & 4 & 4 & 5 & 5 & $3,33 \pm 1,15$ & 40 & 0,02 & Ac \\
\hline $\begin{array}{l}\text { Corvus albus Muller, } 1776 \\
\text { Sturnidae }\end{array}$ & $\mathrm{R}$ & f. & 25 & 23 & 40 & 16 & 33 & 14 & 13 & 10 & 19 & 12 & 11 & 15 & 40 & $19,25 \pm 9,38$ & 231 & 0,13 & Ac \\
\hline $\begin{array}{l}\text { Lamprotornis cupreocauda (Hartlaub, 1857) NT } \\
\text { A05 }\end{array}$ & $\mathrm{R}$ & $\mathrm{F}$ & & & & & & & & & & & & & & & & & \\
\hline Lamprotornis splendidus (Vieillot, 1822) & $\mathrm{R}$ & $\mathrm{F}$ & 355 & 111 & 325 & 511 & 354 & 86 & 130 & 175 & 233 & 354 & 214 & 168 & 511 & $\begin{array}{c}251,33 \pm \\
128,25\end{array}$ & 3016 & 1,73 & $\mathrm{R}$ \\
\hline Cinnyricinclus leucogaster (Boddaert, 1783) & M & f. & 280 & 284 & 167 & 0 & 0 & 0 & 0 & 122 & 82 & 339 & $\begin{array}{c}189 \\
4\end{array}$ & $\begin{array}{c}221 \\
8\end{array}$ & $\begin{array}{c}221 \\
8\end{array}$ & $\begin{array}{c}448,83 \pm \\
763,53\end{array}$ & 5386 & 3,08 & $\mathrm{R}$ \\
\hline Passeridae & & & & & & & & & & & & & & & & & & & \\
\hline Passer domesticus (Linné, 1758) & $\mathrm{R}$ & f. & 0 & 0 & 0 & 0 & 0 & 0 & 0 & 0 & 0 & 1 & 0 & 0 & 1 & $0,08 \pm 0,29$ & 1 & 0 & Ac \\
\hline $\begin{array}{l}\text { Passer griseus (Vieillot, 1817) } \\
\text { Ploceidae }\end{array}$ & $\mathrm{R}$ & f. & 16 & 24 & 28 & 12 & 17 & 11 & 9 & 11 & 13 & 15 & 13 & 13 & 28 & $15,17 \pm 5,59$ & 182 & 0,1 & Ac \\
\hline Ploceus pelzelni (Hartlaub, 1887) & $\mathrm{R}$ & f. & 5 & 10 & 7 & 18 & 9 & 11 & 9 & 9 & 12 & 8 & 13 & 8 & 18 & $9,92 \pm 3,34$ & 119 & 0,07 & Ac \\
\hline Ploceus nigricollis (Vieillot, 1805) & $\mathrm{R}$ & f. & 26 & 31 & 55 & 29 & 24 & 35 & 24 & 27 & 26 & 16 & 15 & 37 & 55 & $28,75 \pm 10,52$ & 345 & 0,2 & $\mathrm{Ra}$ \\
\hline Ploceus aurantius (Vieillot, 1805) A05 & $\mathrm{R}$ & f. & 78 & 49 & 103 & 132 & 178 & 100 & 90 & 107 & 71 & 64 & 74 & 76 & 178 & $93,5 \pm 34,68$ & 1122 & 0,64 & $\mathrm{Ra}$ \\
\hline Ploceus nigerrimus Vieillot, 1819 A05 & $\mathrm{R}$ & f. & 182 & 226 & 133 & 478 & 457 & 331 & 174 & 405 & 432 & 419 & 334 & 208 & 478 & $\begin{array}{c}314,92 \pm \\
124,32\end{array}$ & 3779 & 2,16 & $\mathrm{R}$ \\
\hline Ploceus cucullatus (Muller, 1776) & $\mathrm{R}$ & f. & $\begin{array}{c}278 \\
0\end{array}$ & $\begin{array}{c}251 \\
2\end{array}$ & 403 & 217 & 308 & 957 & $\begin{array}{c}332 \\
4\end{array}$ & 5070 & $\begin{array}{c}1010 \\
6\end{array}$ & $\begin{array}{c}960 \\
8\end{array}$ & $\begin{array}{c}565 \\
0\end{array}$ & $\begin{array}{c}591 \\
4\end{array}$ & $\begin{array}{c}101 \\
06\end{array}$ & $\begin{array}{c}3904,08 \pm \\
3443,74\end{array}$ & $\begin{array}{c}4684 \\
9\end{array}$ & 26,8 & $\mathrm{D}$ \\
\hline Malimbus nitens (Gray, 1831) A05 & $\mathrm{R}$ & $\mathrm{F}$ & 2 & 3 & 3 & 1 & 0 & 0 & 0 & 4 & 2 & 0 & 3 & 2 & 4 & $1,67 \pm 1,44$ & 20 & 0,01 & Ac \\
\hline Malimbus scutatus (Cassin, 1849) A05 & $\mathrm{R}$ & $\mathrm{FF}$ & 9 & 8 & 5 & 4 & 7 & 15 & 7 & 8 & 11 & 9 & 12 & 10 & 15 & $8,75 \pm 3,02$ & 105 & 0,06 & Ac \\
\hline Quelea erythrops (Hartlaub, 1848) & M & f. & 100 & 450 & 900 & 180 & 125 & 8440 & 142 & 1208 & 550 & 100 & 0 & 0 & 142 & $3323,75 \pm$ & 3988 & 22,82 & $D$ \\
\hline
\end{tabular}




\begin{tabular}{|c|c|c|c|c|c|c|c|c|c|c|c|c|c|c|c|c|c|c|c|}
\hline ORDRES /Familles/Espèces & Bio & $\mathrm{Hab}$ & Jan & Fév & Mars & Avr & Mai & Juin & Juil & Août & Sept & Oct & Nov & Déc & IPA & $\begin{array}{c}\text { Moy } \\
\text { Men士Ec-Ty }\end{array}$ & EC & $\mathrm{Fr}$ & IA \\
\hline Euplectes afer (J. F. Gmelin, 1789) & $\mathrm{R}$ & f. & 461 & 425 & 170 & $\begin{array}{c}3 \\
176\end{array}$ & $\begin{array}{c}2 \\
395\end{array}$ & 209 & $\begin{array}{c}10 \\
241\end{array}$ & $\begin{array}{c}0 \\
212\end{array}$ & 257 & 323 & 664 & 975 & $\begin{array}{c}10 \\
975\end{array}$ & $\begin{array}{c}5158,13 \\
375,67 \pm 238,2\end{array}$ & $\begin{array}{c}5 \\
4508\end{array}$ & 2,58 & $\mathrm{R}$ \\
\hline $\begin{array}{l}\text { Euplectes macrourus (J. F. Gmelin, 1789) } \\
\text { Estrildidae }\end{array}$ & $\mathrm{R}$ & f. & 170 & 103 & 57 & 79 & 53 & 50 & 75 & 70 & 72 & 68 & 77 & 108 & 170 & $81,83 \pm 32,85$ & 982 & 0,56 & $\mathrm{Ra}$ \\
\hline Nigrita canicapillus (Strickland, 1841) & $\mathrm{R}$ & $\mathrm{F}$ & 7 & 8 & 7 & 5 & 7 & 4 & 8 & 8 & 9 & 6 & 10 & 6 & 10 & $7,08 \pm 1,68$ & 85 & 0,05 & Ac \\
\hline Nigrita bicolor (Hartlaub, 1844) A05 & $\mathrm{R}$ & FF & 7 & 7 & 9 & 6 & 6 & 4 & 5 & 6 & 6 & 6 & 9 & 9 & 9 & $6,67 \pm 1,61$ & 80 & 0,05 & Ac \\
\hline Pyrenestes ostrinus (Vieillot, 1805) & $\mathrm{R}$ & $\mathrm{F}$ & 9 & 10 & 15 & 7 & 20 & 27 & 24 & 19 & 12 & 9 & 19 & 20 & 27 & $15,92 \pm 6,52$ & 191 & 0,11 & Ac \\
\hline Spermophaga haematina (Vieillot, 1807) A05 & $\mathrm{R}$ & $\mathrm{F}$ & 6 & 3 & 2 & 5 & 3 & 3 & 3 & 2 & 8 & 9 & 7 & 7 & 9 & $4,83 \pm 2,48$ & 58 & 0,03 & Ac \\
\hline Estrilda melpoda (Vieillot, 1817) & $\mathrm{R}$ & f. & 506 & 477 & 393 & 269 & 519 & 305 & 380 & 301 & 249 & 216 & 400 & 628 & 628 & $386,92 \pm 125,9$ & 4643 & 2,66 & $\mathrm{R}$ \\
\hline Sporaeginthus subflavus (Vieillot, 1819) & $\mathrm{R}$ & f. & 13 & 7 & 0 & 2 & 0 & 0 & 4 & 2 & 1 & 8 & 1 & 2 & 13 & $3,33 \pm 4,03$ & 40 & 0,02 & Ac \\
\hline Ortygospiza atricollis (Vieillot, 1817) & $\mathrm{R}$ & f. & 24 & 4 & 4 & 2 & 8 & 0 & 1 & 12 & 74 & 41 & 4 & 10 & 74 & $15,33 \pm 21,89$ & 184 & 0,11 & Ac \\
\hline Spermestes cucullata (Swainson, 1837) & $\mathrm{R}$ & f. & $\begin{array}{c}171 \\
3\end{array}$ & $\begin{array}{c}132 \\
0\end{array}$ & 683 & 574 & 456 & 329 & 643 & 934 & 1956 & $\begin{array}{c}175 \\
0\end{array}$ & $\begin{array}{c}133 \\
5\end{array}$ & $\begin{array}{c}159 \\
9\end{array}$ & $\begin{array}{c}195 \\
6\end{array}$ & $\begin{array}{c}1107,67 \pm \\
570,41\end{array}$ & $\begin{array}{c}1329 \\
2\end{array}$ & 7,6 & D \\
\hline Spermestes bicolor (Fraser, 1843) & $\mathrm{R}$ & f. & 165 & 112 & 95 & 85 & 62 & 38 & 25 & 54 & 452 & 164 & 114 & 115 & 452 & $\begin{array}{c}123,42 \pm \\
112,61\end{array}$ & 1481 & 0,85 & $\mathrm{Ra}$ \\
\hline Spermestes fringilloides (Lafresnaye, 1835) & $\mathrm{R}$ & f. & 380 & 318 & 331 & 191 & 124 & 55 & 78 & 83 & 166 & 138 & 277 & 285 & 380 & $\begin{array}{c}202,17 \pm \\
111,62\end{array}$ & 2426 & 1,39 & $\mathrm{R}$ \\
\hline Viduidae & & & & & & & & & & & & & & & & & & & \\
\hline Vidua macroura (Pallas, 1764) & $\mathrm{R}$ & f. & 83 & 57 & 63 & 66 & 48 & 59 & 52 & 107 & 289 & 255 & 127 & 56 & 289 & $105,17 \pm 81,74$ & 1262 & 0,72 & $\mathrm{Ra}$ \\
\hline Totaux & & & $\begin{array}{c}117 \\
68\end{array}$ & $\begin{array}{c}157 \\
72 \\
\end{array}$ & 6776 & $\begin{array}{c}746 \\
5\end{array}$ & $\begin{array}{c}756 \\
3\end{array}$ & $\begin{array}{c}1336 \\
1\end{array}$ & $\begin{array}{c}218 \\
61\end{array}$ & $\begin{array}{c}2371 \\
7\end{array}$ & $\begin{array}{c}1904 \\
9\end{array}$ & $\begin{array}{c}170 \\
57\end{array}$ & $\begin{array}{c}148 \\
04\end{array}$ & $\begin{array}{c}156 \\
06\end{array}$ & $\begin{array}{c}469 \\
00\end{array}$ & $\begin{array}{c}14566,58 \pm \\
5520,9\end{array}$ & $\begin{array}{c}1747 \\
99\end{array}$ & 100 & \\
\hline
\end{tabular}

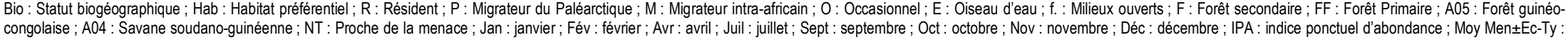

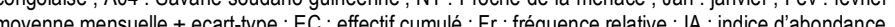




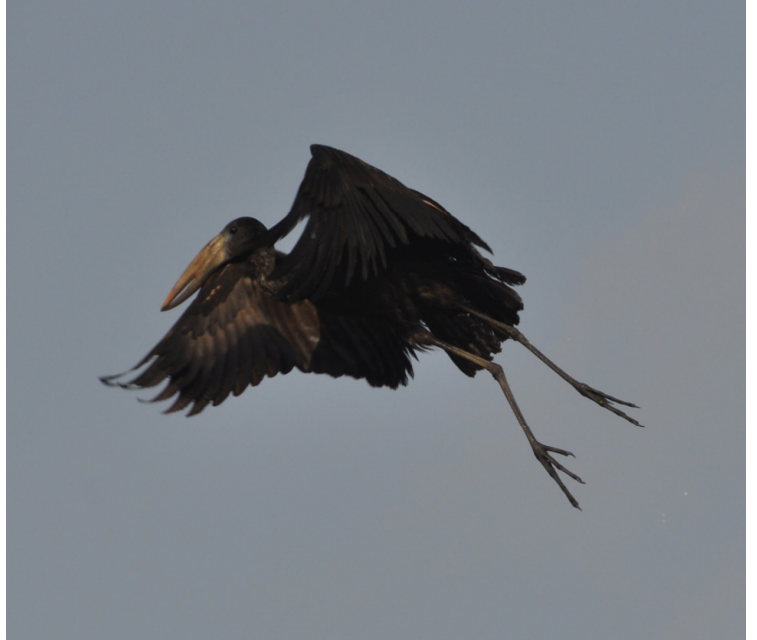

Figure 1a. Bec ouvert africain Anastomus lamelligerus

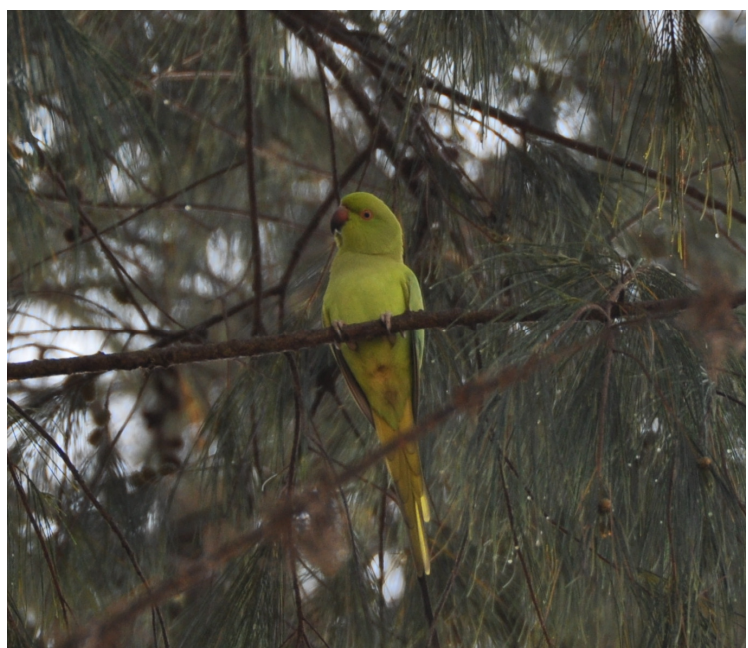

Figure 1c. Perruche à collier Psittacula krameri

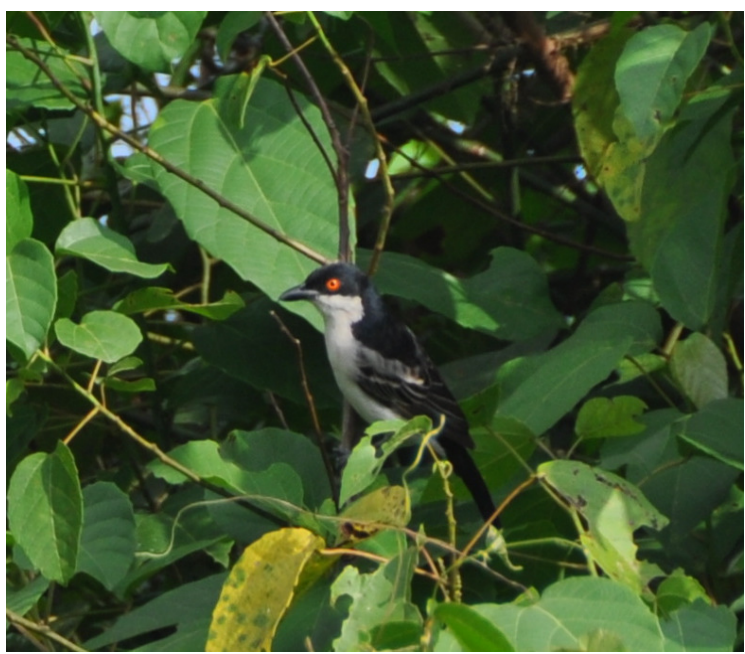

Figure 1e. Cubla de Gambie Dryoscopus gambensis

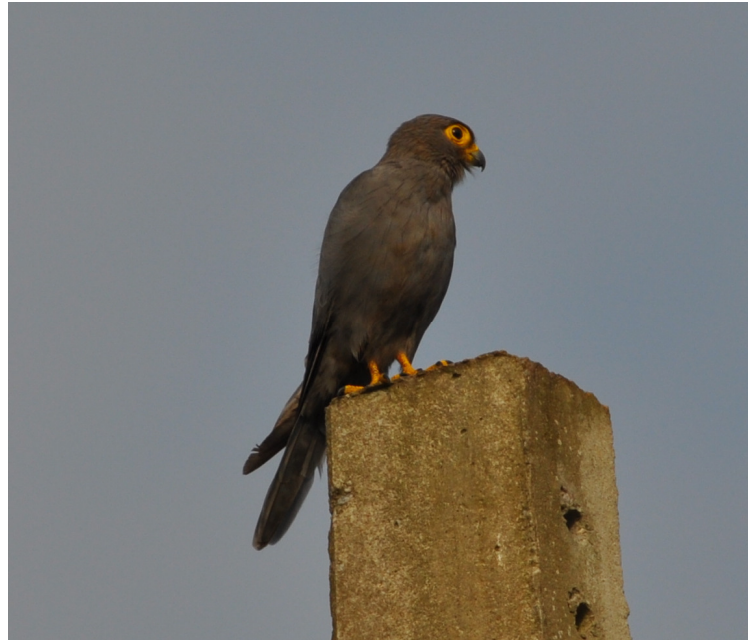

Figure 1b. Faucon ardoisé Falco ardosiaceus

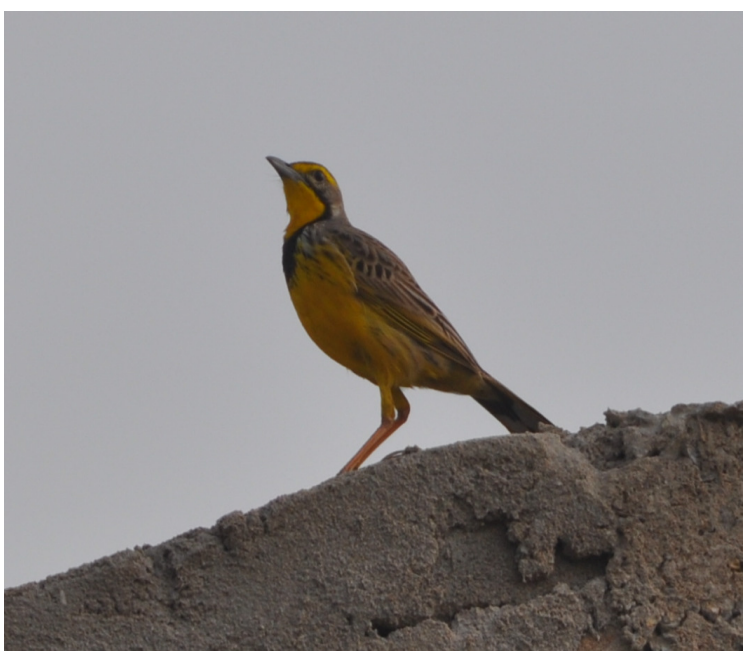

Figure 1d. Sentinelle à collier Macronyx croceus

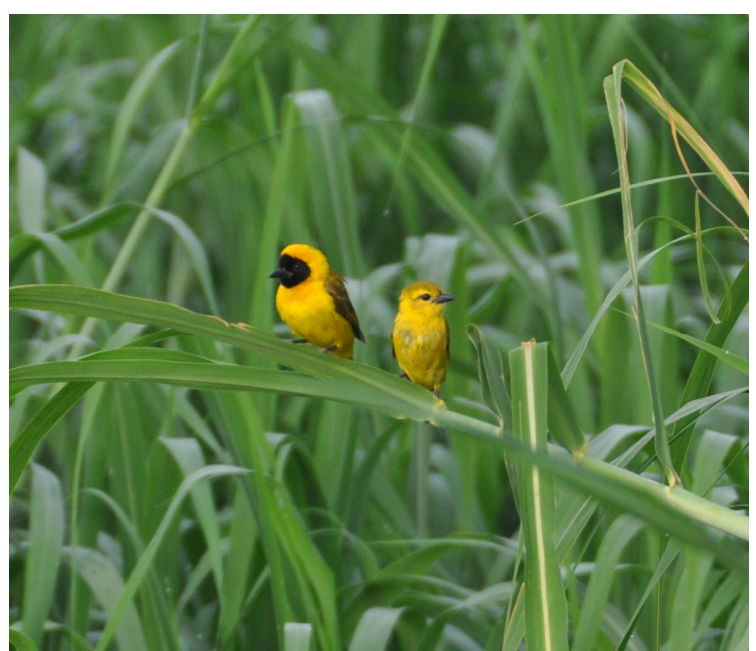

Figure 1f. Tisserin de Pelzeln Ploceus pelzelni

Figure 1 : Images de quelques espèces d'oiseaux rencontrées dans la zone humide d'importance internationale de Grand-Bassam

Caractérisation qualitative du peuplement : Selon le statut biogéographique, le peuplement est dominé par les espèces résidentes avec 145 espèces soit $66,51 \%$ du peuplement. Les espèces migratrices 
représentent $19,73 \%$ du peuplement avec 43 espèces. Les espèces à statut mixte c'est-à-dire résidentes et migratrices avec 30 espèces représentent $13,76 \%$ du peuplement. En ce qui concerne l'habitat préférentiel, les espèces des milieux ouverts dominent avec 93 espèces soit $42,66 \%$ du peuplement. Les espèces des zones humides ainsi que celles des forêts secondaires représentent 22,93\% du peuplement avec 50 espèces. Les espèces des forêts primaires avec 25 espèces soit $11,46 \%$ du peuplement sont moins représentées.

Espèces d'intérêt pour la conservation: Trois espèces sont inscrites sur la liste rouge de I'UICN : une espèce vulnérable le perroquet jaco Psittacus erithacus Linné, 1758 et deux espèces proches de menace la grue couronnée Balearica pavonina (Linné, 1758) et le choucador à queue bronzée Lamprotornis cupreocauda (Hartlaub, 1857). Cinquante-trois espèces du biome de la forêt Guinéocongolaise (A05) et trois espèces du biome des savanes Soudano-guinéennes (A04) sont présentes dans la zone de riziculture et ses environs.

Structure du peuplement d'Oiseaux: L'analyse du tableau 1 revèle un effectif cumulé de 174799 spécimens d'Oiseaux avec un effectif moyen mensuel de 14566,58 $\pm 5520,9$ individus et une charge avifaunistique (IPA) de 46900 individus d'Oiseaux. L'indice de diversité de Shannon $\left(H^{\prime}\right)$ de la zone de riziculture est de 2,85 avec une équitabilité (J) de 0,54. Le peuplement d'Oiseaux de la zone de riziculture de Grand-Bassam est constitué de trois espèces dominantes, 12 espèces régulières, 25 espèces rares et 158 espèces accidentelles. Le tisserin gendarme Ploceus cucullatus (Muller, 1776), le travailleur à tête rouge Quelea erythrops (Hartlaub, 1848) et le capucin nonnette Spermestes cucullata (Swainson, 1837) sont les espèces les plus abondantes et contribuent à $57,22 \%$ du peuplement. Les espèces régulières représentent $27,21 \%$ du peuplement et les autres (espèces rares et accidentelles) représentent seulement $15,58 \%$ du peuplement.

Variation mensuelle du peuplement: La courbe réalisée à partir de la matrice "effectif du peuplement/mois/année" montre une variation du peuplement des Oiseaux au cours du temps (Figure 2). En effet, l'analyse de variance montre une différence hautement significative entre les effectifs mensuels des Oiseaux (ddl $=12 ; \mathrm{F}=3,51 ; \mathrm{p}$ $<0,001$ ). Les fluctuations du peuplement au cours des deux années d'observation montrent des similitudes qui se résument en trois flux principaux. Une croissance plus ou moins régulière $d u$ peuplement s'observe durant la période d'avril à juillet et atteint le maximum au mois de juillet. De juillet jusqu'en fin novembre, le peuplement décline cette fois. Mais à la première moitié de la grande saison sèche, ce peuplement augmente légèrement pour enfin baisser à la seconde moitié de cette même saison.

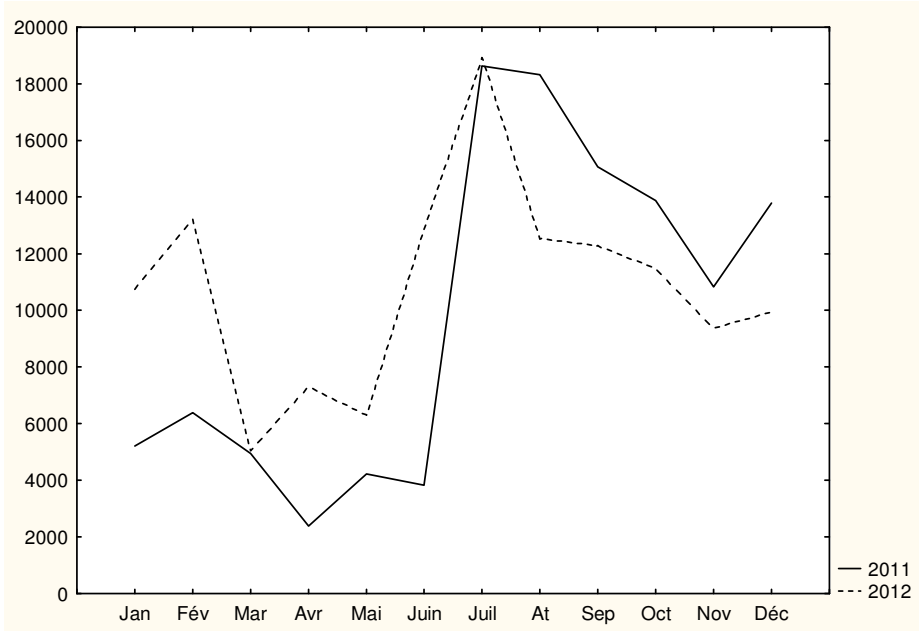

Figure 2 : Graphique présentant les variations mensuelles du peuplement d'Oiseau.

\section{DISCUSSION}

Cette étude menée sur deux cycles annuels confirme la richesse de l'avifaune de la zone de riziculture de Grand-Bassam. Avec ses 218 espèces, la zone de riziculture regroupe plus de $80 \%$ des espèces rencontrées dans toute la zone humide de GrandBassam. Par comparaison aux travaux antérieurs (Yaokokoré-Béibro et al., 2010; Odoukpé, 2010; Gueye, 2013) qui mentionnent 229 espèces 
d'Oiseaux pour la zone humide de Grand-Bassam,la présente étude révèle 49 espèces nouvellement observées. Les observations les plus spectaculaires sont celles qui font mention d'espèces identifiées pour la première fois dans le sud forestier ivoirien. Les espèces concernées sont la grue couronnée Balearica pavonina, le barbican à poitrine rouge Lybius dubius (Gmelin, 1788), le pic goertan Dendropicos goertae (Statius Muller, 1776) et le bengali zébré Sporaeginthus subflavus (Vieillot, 1819). La liste des espèces de la zone humide de Grand-Bassam s'estime désormais à 278 espèces appartenant à 60 familles et 20 ordres. Deux espèces d'entre elles, le faucon crécerellette Falco naumanni et le perroquet jaco Psittacus erithacus, sont inscrites comme des espèces vulnérables sur la liste rouge de I'UICN (2012). Sept espèces d'entre elles sont proches de la menace dans la liste rouge de I'UICN (Yaokokoré-Béibro et al., 2010 ; Gueye, 2013). II s'agit du puffin fuligineux Puffinus griseus (Gmelin, 1789), de la barge à queue noire Limosa limosa (Linné, 1758), du courlis cendré Numenius arquata (Linné, 1758), de la sterne des baleiniers Sterna balaenarum (Strickland, 1857), de la grue couronnée Balearica pavonina, du calao à joues brunes Bycanistes cylindricus (Temminck, 1831) et du choucador à queue bronzée Lamprotornis cupreocauda. La zone humide de Grand-Bassam compte à présent soixante espèces endémiques du biome de la forêt guinéo-congolaise et trois espèces du biome des savanes soudano-guinéennes (Fishpool et Evans, 2001). La forte présence d'Oiseaux des milieux ouverts est la preuve d'une anthropisation de plus en plus prononcée de la zone liée au développement de la ville et aux activités agricoles (Yaokokoré-Béibro et al., 2010). Les quelques vestiges de forêts qu'on y trouve sont

\section{CONCLUSION}

Cette étude contribue à une connaissance approfondie de l'avifaune de la zone humide de Grand-Bassam et par extension de celle du littoral ivoirien. C'est une zone favorable aux communautés d'Oiseaux qui y trouvent des ressources alimentaires abondantes et diversifiées déclenchant ainsi leur instinct au grégarisme. En matière d'études

\section{REMERCIEMENTS}

Nos remerciements s'adressent particulièrement à Mme Koné Solange et M. Gueye Monnoin Frédéric pour leur soutien sans faille lors des rélevés de terrain à Grand-Bassam. Nous sommes reconnaissants à $\mathrm{M}$. Kouamé et à l'ensemble des fortement modifiés et dégradés par les activités des riverains. Pourtant plus d'une vingtaine d'espèces mentionnées sont caractérisées par YaokokoréBéibro (2001) et Bennun et al. (1996) comme étant des espèces sténotypiques de forêt. Aussi, certaines de ces espèces, Psittacus erithacus, Pogoniulus bilineatus, Nigrita bicolor ont été observées dans des zones moins boisées et souvent même en milieux urbanisés. L'adaptation à d'autres types de milieux, face à la dégradation pressante et persistante des milieux naturels, pourrait bien expliquer ces observations. II serait donc convenable de reviser les caractérisations des espèces d'Oiseaux de Côte d'Ivoire en ce qui est de leur habitat préférentiel. Cette pléiade d'individus du peuplement d'Oiseaux de la zone de riziculture de Grand-Bassam illustre bien l'influence des rizières sur la structure des communautés d'Oiseaux. Les cultures de riz permettent aux espèces d'Oiseaux de s'alimenter en masse (Tréca, 1989) parce qu'ils sont généralement assurés de trouver la nourriture en quantité inépuisable. Ce peuplement d'Oiseaux s'amplifie à la fin de la grande saison pluvieuse et dans une moindre mesure en début de la grande saison sèche parce que la majeure partie des surfaces mises en valeur arrive en phase de reproduction. C'est une communauté largement dominée par trois populations d'espèces grégaires que sont le tisserin gendarme, le travailleur à tête rouge et le capucin nonnette. Le tisserin gendarme et le capucin nonnette s'y reproduisent et prolifèrent rapidement puisqu'ils ne sont plus limités par la nourriture disponible pour élever les jeunes. Par contre, les populations de travailleur à tête rouge ne s'y reproduisent pas mais forment les plus grandes bandes lorsqu'elles sont présentes dans la zone.

ornithologiques, la zone humide de Grand-Bassam fait partie des milieux les plus prospectés en Côte d'Ivoire. Toutefois, un suivi régulier des différentes populations d'Oiseaux présentes dans toute la zone humide permettra d'établir un système de gestion durable de l'avifaune.

paysans de la zone de riziculture de Grand-Bassam pour leur coopération et leur disponibilité à nous venir en aide chaque fois qu'il était nécessaire pendant la période de récolte des données. 


\section{RÉFÉRENCES BIBLIOGRAPHIQUES}

Benun L., Dranzoa C., Pomeroy D., 1996. The forest birds of Kenyan and Uganda. Journal of East African Natural History, 85 : 23-48.

Bibby C. J., Burgess N. D., Hill D. A., 1992. Birds census techniques. BTO/RSPB. Academic Press, London, UK, $257 \mathrm{pp}$.

Borrow N. et Demey R., 2001. Birds of Western Africa. London, Grande Bretagne, HELM Identification guides, 800p.

Borrow N. et Demey R., 2008. Guide des Oiseaux de l'Afrique de l'Ouest. Paris, Delachaux et Niestlé, 510p.

Brooks T., Andriamaro L., Gereau R., Hawkins F., Howell II P. P., Luke Q., Matiku P., McKnight M. W., Msuya C., Mugo R., Rabarison H., Rakotobe Z. L., Randrianasolo H., 2007. Objectifs et priorités pour la conservation des Oiseaux et de la biodiversité d'Afrique. Ostrich 78 (2) : 115-234.

Brou Y., 1997. Analyse et dynamique de la pluviométrie en milieu forestier ivoirien. Thèse de Doctorat 3ème cycle. Université de Cocody Abidjan, $200 \mathrm{p}$.

Secrétariat de la convention de Ramsar, 2006. Le manuel de la convention de Ramsar : Guide de la convention sur les zones humides (Ramsar, Iran, 1971), 4 è édition. Secrétariat de la convention de Ramsar, Gland, Suisse, $124 p$.

Secrétariat de la convention de Ramsar, 2010. La liste des zones humides d'importance internationale. Secrétariat de la convention de Ramsar, Gland, Suisse, 43p.

Chappuis C., 2000. African Bird Sounds. Birds of North, West and Central Africa. Livrette et $15 \mathrm{CD}$. Société d'Études Ornithologiques de France, Paris.

Fishpool L. D. C. et Evans M. I., 2001. Important Bird Areas in Africa and Associated Islands. CDROM. Cambridge,BirdLife International.

Fotso R. C., 1994. Dynamique des peuplements d'Oiseaux dans les séries écologiques de la région de Yaoundé (Sud Cameroun). Thèse de Doctorat es Sciences. Université catholique de Leuvens, 142p.

Gueye M. F., 2013. Inventaire ornithologique d'un milieu urbain côtier de Côte d'lvoire : cas de la ville de Grand-Bassam. DEA Écologie Tropicale, Université Félix HouphouëtBoigny, Abidjan, $54 \mathrm{p}$.

Guillaumet J.-L. et Adjanohoun E., 1971. La végétation de la Côte d'Ivoire. In : Le milieu naturel de la Côte d'Ivoire. Paris, Mémoire ORSTOM, 50 : 157-263.
James A., Gaston K., Balmford A., 1999. Balancing the Earth's accounts. Nature $401: 323-324$.

Lachenaud O., 2006a. Les Oiseaux du parc national du Banco et de la forêt classée de l'Anguédédou, Côte d'Ivoire. Malimbus 28 (2) : 107-133.

Lachenaud O., 2006b. Les Oiseaux de la région de Sassandra, Côte d'Ivoire. Malimbus, 28 (1) : 18-34.

Odoukpé K. S. G., 2010. Avifaune d'une Zone de Riziculture dans la Zone Humide d'Importance Internationale de Grand Bassam. DEA Écologie Tropicale, Université de Cocody-Abidjan, $56 \mathrm{p}$.

Ramade F., 2005. Éléments d'écologie: écologie appliquée. Paris, DUNOD, 863p

Serle W. \& Morel G. J., 1993. Les Oiseaux de l'Ouest africain. Lausanne, Delachaux et Niestlé, $331 p$.

Thiollay J. M., 1986. Structure comparée du peuplement avien des trois sites de forêt primaire en Guyane. La terre et la vie, 41: 59-105.

Tréca B., 1989. Les risques de dégâts d'Oiseaux sur les rizières sahéliennes. In : Eldin M. \& Milleville P. (Eds). Le risque en agriculture. Paris, France, ORSTOM : 167-175.

UICN, 2012. IUCN red list of threatened species. Version 2012.2.

Waltert M., Yaokokoré-Béibro K. H., Mühlenberg M., Waitkuwait W. E., 1999. Preliminary checklist of the birds of the Bossématié area, Ivory Coast. Malimbus 21 (2) : 93-109.

Yaokokoré Béibro K. H. et Ellenberg H., 2000. A contribution to the study of the avifauna of Bossematie Forest (Abengourou/Côte d'Ivoire). The future of the Bossematie Forest : current status. Ostrich 71 (1\&2) : 292-294.

Yaokokoré-Béibro K. H., 2001. Avifaune des forêts classées de l'Est de la Côte d'lvoire: données sur l'écologie des espèces et effet de la déforestation sur les peuplements. Cas des forêts classées de la Béki et de la Bossématié (Abengourou). Thèse de Doctorat, Université de Cocody, 245p.

Yaokokoré-Béibro K. H., N'Douba V. et Comité de Ramsar, 2005. Fiche descriptive sur les zones humides Ramsar (FDR), 17p.

Yaokokoré-Béibro K. H., 2010a. Diversité avifaunique de la forêt classée de la Besso, Sud-Est de la Côte d'Ivoire. Sciences \& Nature Vol.7 $\mathrm{N}^{\circ} 2: 207-219$. 
Yaokokoré-Béibro K. H., 2010b. Oiseaux du Parc National des lles Ehotilé, sud-est Côte d'Ivoire. Malimbus $32: 89-102$.

Yaokokoré-Béibro K. H., N'guessan A. M., Odoukpé K. S. G., Zouzou E. J., N'douba V., Kouassi K. P., 2010. Premières données sur les Oiseaux de la zone humide d'importance internationale de Grand-Bassam (Côte d'Ivoire). International Journal of Biological and Chemical Sciences 4 (6) : 2169-2180. 